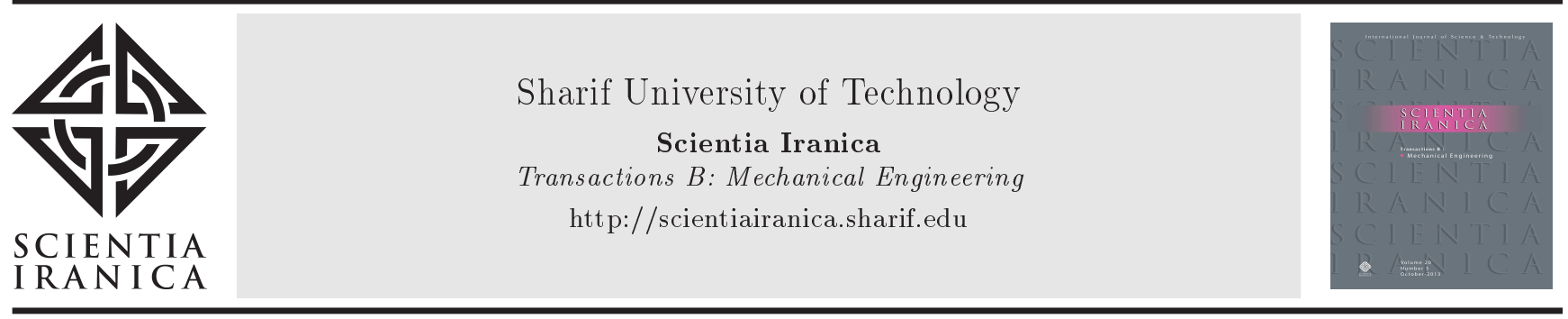

\title{
Tracking control and vibration reduction of flexible cable-suspended parallel robots using a robust input shaper
}

\author{
M.H. Korayem*, M. Yousefzadeh, and S. Manteghi \\ Robotic Research Laboratory, Center of Excellence in Experimental Solid Mechanics and Dynamics, School of Mechanical \\ Engineering, Iran University of Science and Technology, Tehran, P.O. Box 13114-16846, Iran.
}

Received 12 March 2016; received in revised form 28 September 2016; accepted 7 January 2017

KEYWORDS
Cable-suspended
parallel robots;
Gibbs-appel;
Singular perturbation;
Input shaping;
Feedback
linearization.

\section{Introduction}

Cable-suspended parallel robot is a type of parallel robot, where the links are replaced with cables connecting the actuators (electric motors) to the endeffector. In comparison with other parallel robots, they benefit from features such as larger workspace, lower

*. Corresponding author. Tel.: +982173912904

E-mail addresses: hkorayem@iust.ac.ir (M.H. Korayem);

myousefzafeh@iust.ac.ir (M. Yousefzadeh);

soleimanmantegi@gmail.com (S. Manteghi)

doi: $10.24200 /$ sci.2017.4500 manufacturing technology, higher ratio of the carrying load per robot's moving weight, and lower repairs and maintenance expenses. Due to the high flexibility of cables compared to the links, the system response is vibratory.

The end-effector vibration due to the transversal vibration of the cables is negligible in comparison with that due to the axial vibration of the cables [1]. To improve the system response, the state variables added to the system because of the axial vibrations of the cables should be considered in the controller. In this regard, Korayem et al. described the dynamic equations of a 6 DOF cable-suspended robot with elastic joints and controlled the end-effector employing a robust feedback 
linearization method [2]. In another study, they incorporated the tension obtained from PDE solution of the vibrational cables into the SMC controller in order to compensate for flexibility uncertainties [3].

Control of flexible cable robots can also be accomplished using the measurements of the cables tension. Laroche et al. modeled the cables of a 3DOF cable robot, with a point mass end-effector, as springs and controlled the robot by $H_{\infty}$ method. They used the feedback from the end-effector pose and the mean tension of the cables in the controller [4]. Meunier et al. derived the equations of the reflector of a large radio telescope by discretizing the cables utilizing lumped mass elements, where springs and dampers were placed between them [5]. They employed a cascade approach, including cable tension control using $H_{\infty}$ method in the inner loop, and end-effector pose control using PID computed-torque method in the outer loop. Lumped-mass method was also utilized by Caverly et al. in modeling the cables of a 2DOF planar cable-driven robot [6]. They incorporated the change in the cable stiffness when the cables were wounded around the winches. In this research, passivity-based control method was employed. Due to the flexibility of the long-span cables in the $500 \mathrm{~m}$ Aperture Spherical radio Telescope (FAST), the receiver may encounter a possible vibration induced by the wind disturbance. Thus, a rigid Stewart platform was used to compensate for the positioning error [7]. Nevertheless, when the mass and inertia of the receiver are not negligible in comparison with the base of the Stewart system, the dynamic interaction becomes significant. This, in turn, challenges the compensating control of the rigid mechanism [8].

In order to control over-constrained cable robots, Khosravi and Taghirad modeled the cables as spring. They showed that asymptotic stability of the closed loop system can be guaranteed using a composite control comprising a rigid model based controller and a PD term, proportional to the time derivative of the tension [9]. A survey of the control of flexible joint robots is provided in [10], where different approaches such as composite control and the concept of integral manifold are compared.

In all of the above works, to prevent vibrations, another feedback in addition to the actuators state was required. However, if an approximation of the system's natural frequency and damping parameters is available, filtering methods such as input shaping can help prevent the excitation of natural modes and reduce the vibrations. Advantages of input shaping methods over conventional filters, which are designed in frequency domain, are shown in [11].

Vibration reduction of flexible system using input shaping is usually accompanied by a closed loop controller, in order to compensate for uncertainties and disturbance rejection. In this regard, Luo et al. employed a perturbation method to separate slow and fast dynamics of a $3 \mathrm{R}$ planar parallel manipulator [12]. They used a composite controller comprising a feedback linearization method for the control of slow dynamics and Zero Vibration Derivative (ZVD) input shaping for vibration reduction, caused by elastic links. Hu applied a Variable Structure Controller (VSC), an estimator, and an input shaper for the control of a spacecraft with elastic wings [13]. Active vibration control of a 3P2R planar parallel manipulator was accomplished by Zhang et al. [14]. The controller was a combination of a VSC method for motion tracking of the end-effector and a strain rate feedback control for suppressing the vibration of the elastic linkages. They also investigated the effectiveness of combining a PD computed-torque control with the ZVD input shaper for suppressing the residual vibration of the mechanism [15].

Extra Insensitive (EI) input shaper was used to address the vibration reduction at the end of the point-to-point movement of a flexible delta robot [16]. The damping ratio and natural frequency required in the input shaper were obtained from a look-up table, considering the position of the end-effector in the workspace. Li et al. proposed a vibration control scheme for a two-link flexible joint manipulator mounted on a Hexapod active manipulator [17]. The controller was composed of a linear extended state observer, a linear feedback controller, and an adaptive law. The ZVDD input shaper was used to alleviate the vibration induced by input motions, while the closed loop controller compensated for the unknown external disturbances.

A number of studies have been devoted to command shaping to address the payload oscillation in cranes. Blackburn studied the performance of different input shaping methods in vibration suppression of construction cranes [18]. Sway control of the endeffector of a 2-cable robot using ZV and ZVD input shapers was proposed by Park et al. In this work, natural frequency for any point of the workspace was obtained from system linearization around the associated equilibrium point [19]. Anti-sway control of a gantry crane, modeled as a double pendulum, was also accomplished using Specified Insensitivity (SI) input shaping method in [20]. Garrido et al. controlled the swinging of a crane by input shaping, where the disturbances were obtained using two cableslope measuring sensors and cancelled by applying a specific input at a specific time [21]. The marine cranes suffer from swinging problem, particularly in the presence of sea disturbances. Elbadawy and Shehata proposed a closed-loop PID input shaper for point-topoint motion without swinging [22]. In the experiment, they mounted the crane on a Stewart manipulator to simulate the sea disturbances. 
One of the challenges encountered by the cablesuspended robots is the possibility of zero tension in the cables due to the vibration caused by the flexibility. In the point-to-point movement command, the initial error is large and if it is accompanied by high gains, the controller calculates a large control effort at the beginning of the path, leading to severe tension oscillation and possibility of zero tension in the cables. Consequently, there is a time limitation in reaching the desired point, as a result of the maximum gain constraint. Similarly, in trajectory tracking, severe trajectory change can also result in tension and endeffector vibrations. If tension oscillations of the cables are suppressed, the system can reach the desired point at a shorter time.

Using all the states introduced by the system flexibility in the controller can be difficult due to the numerous required sensors with different types and performances, and increase in the controller and estimator computations on the processor. In this study, vibration of the end-effector and the cable tension is reduced by robust input shaping of the desired input. In the case of high cable flexibility, this method noticeably reduces the vibration of the end-effector. In the low cables flexibility condition, the contribution of input shaping is in the suppression of the cable tension oscillation, and reduction in the settling time.

This paper is divided into the following sections: In Section 2, the dynamic equations of the 6DOF cable-suspended robot with elastic cables are derived using Gibbs-Appel formulation. Next, stability of the flexible system considering the rigid model based controller is investigated in Section 3. In Section 4, three control approaches are investigated: a) composite control comprising a rigid model based feedback linearization for slow dynamics and a term for damping the vibrations, b) full state feedback linearization, and c) feedback linearization based on the rigid model. These methods are compared based on the computation overhead and required feedbacks for the controller. In Section 5, the formulations related to the multimode robust input shaping are introduced and in Section 6, they are combined with the rigid model based feedback linearization to represent two applications of this approach, i.e., vibration suppression of the end-effector and reducing the settling time. In addition, the efficiency of the proposed approach in vibration reduction is demonstrated by comparing with a composite controller. In Section 7 , the efficiency of the approach is shown by implementing the presented method on a $6 \mathrm{DOF}$ cable-suspended robot, made for experiments in the robotic lab of Iran University of Science and Technology (ICaSbot). The investigation is accomplished by vibration measurement via IMU sensor and frequency analysis.

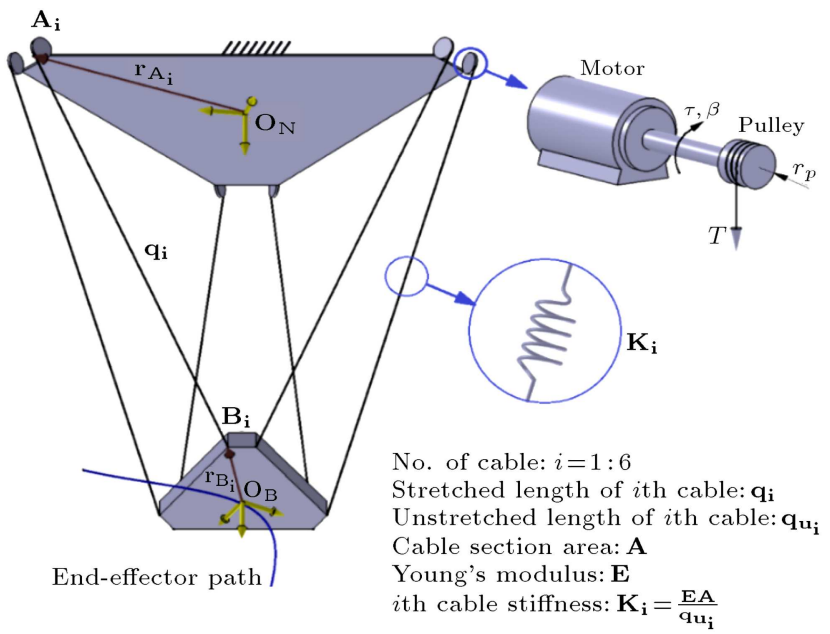

Figure 1. Schematic view of the 6 DOF cable-suspended robot.

\section{Dynamic equations of the robot with elastic cable}

Figure 1 shows the schematic view of the cablesuspended robot studied in this paper. The triangular end-effector of the robot moves by six elastic cables with elasticity $K_{i}$. The center of the coordinate system attached to the end-effector is denoted by $O_{B}$, where the Center of Gravity (C.G.) of the end-effector is located. The position of $O_{B}$ expressed in the inertial frame $N$ is given by $\chi$. The vector $\omega$ shows the angular velocity of the end-effector expressed in the frame attached to the end-effector. The endpoint position of the $i$ th cable on the end-effector is denoted by $\mathbf{r}_{\mathbf{B}_{\mathbf{i}}}$ in the frame attached to the end-effector. The vector $\mathbf{r}_{\mathbf{A}_{\mathbf{i}}}$ indicates the other endpoint position of the $i$ th cable in the inertial frame. The length vector of the $i$ th cable in the inertial frame can be expressed as follows:

$$
\mathbf{q}_{\mathbf{i}}=\chi+{ }^{\mathrm{N}} \mathbf{R}_{\mathrm{B}} \mathbf{r}_{\mathrm{B}_{\mathbf{i}}}-\mathbf{r}_{\mathbf{A}_{\mathbf{i}}},
$$

where ${ }^{\mathbf{N}} \mathbf{R}_{\mathbf{B}}$ is the rotation matrix of the end-effector frame with respect to the inertial frame. Considering $\mathbf{q}_{\mathbf{o}}$ and $\mathbf{q}_{\mathbf{u}}$ as the cable length vectors in the initial and unstretched states, respectively, the following equations can be expressed:

$$
\begin{aligned}
& \mathbf{q}_{\mathbf{u}}=\mathbf{q}_{\mathbf{o}}-r_{p} \boldsymbol{\beta}, \\
& \Delta \mathbf{q}=\mathbf{q}-\mathbf{q}_{\mathbf{u}}, \\
& \dot{\boldsymbol{\beta}}=-\frac{1}{r_{p}} \dot{\mathbf{q}}_{\mathbf{u}}=\frac{1}{r_{p}}(\Delta \dot{\mathbf{q}}-\dot{\mathbf{q}}),
\end{aligned}
$$

where $\boldsymbol{\beta}$ denotes the rotation vector of the drums with radius $r_{p}$. The cables are pulled using the drums on the output shaft of the motors. The velocity vector of the end-effector $\dot{\tilde{\boldsymbol{x}}}$ consisting of the linear velocity 
in the inertial frame and the angular velocity in the end-effector frame is defined as:

$$
\begin{aligned}
& \dot{\tilde{\mathbf{x}}}=\left[\begin{array}{c}
\dot{\chi} \\
\boldsymbol{\omega}
\end{array}\right], \quad \text { where }: \chi=[x, y, z]^{T}, \\
& \boldsymbol{\omega}=\left[\omega_{x}, \omega_{y}, \omega_{z}\right]^{T} .
\end{aligned}
$$

The derivatives of $\mathbf{q}$ relate to the velocity and acceleration vectors of the end-effector as follows:

$$
\begin{aligned}
& \dot{\mathbf{q}}=-r_{p} \mathbf{A}_{\mathbf{1}} \dot{\tilde{\mathbf{x}}} \\
& \ddot{\mathbf{q}}=-r_{p}\left(\mathbf{A}_{\mathbf{1}} \ddot{\tilde{\mathbf{x}}}+\mathbf{A}_{\mathbf{2}}\right),
\end{aligned}
$$

where the matrices $\mathbf{A}_{\mathbf{1}}$ and $\mathbf{A}_{\mathbf{2}}$ are defined in Appendix A. The angular velocity and acceleration of the drums, i.e. $\dot{\boldsymbol{\beta}}$ and $\ddot{\boldsymbol{\beta}}$, can be obtained using Eqs. (2)-(7). They are used in derivation of the dynamic equations. The dynamic modeling is accomplished using GibbsAppel formulation, where the generalized power, the time derivative of the potential energy, the dissipation function, and the Gibbs function of the system are needed. The time derivative of the potential energy due to cable elasticity and gravity can be written as:

$$
\dot{U}_{P}=\boldsymbol{\Delta} \mathbf{q}^{T} \mathbf{K}\left(\mathbf{q}_{\mathbf{u}}\right) \boldsymbol{\Delta} \dot{\mathbf{q}}+\frac{1}{2} \boldsymbol{\Delta} \mathbf{q}^{T} \dot{\mathbf{K}}\left(\mathbf{q}_{\mathbf{u}}\right) \boldsymbol{\Delta} \mathbf{q}-m_{e} g \dot{z}
$$

where:

$$
\mathbf{K}\left(\mathbf{q}_{\mathbf{u}}\right)=\operatorname{diag}\left(\frac{E A}{\mathbf{q}_{\mathbf{u}}}\right),
$$

where $E, A$, and $m_{e}$ denote the Young's modulus of elasticity, cable section area, and the end-effector mass, respectively. By assumption of viscous damping in the cables, the dissipation function is given by:

$$
D=\frac{1}{2} \Delta \dot{\mathbf{q}}^{\mathbf{T}} \mathbf{C}_{\mathbf{d}} \boldsymbol{\Delta} \dot{\mathbf{q}}
$$

where:

$$
\mathbf{C}_{\mathbf{d}}\left(\mathbf{q}_{\mathbf{u}}\right)=\operatorname{diag}\left(\frac{c_{d}}{\mathbf{q}_{\mathbf{u}}}\right)
$$

where $c_{d}$ is the damping coefficient for the unit length of the cables. The Gibbs function of the system can be obtained from the following equation:

$$
S=\frac{1}{2} \ddot{\tilde{\mathbf{x}}}^{T}\left[\mathbf{M}_{\mathbf{e}}\right] \ddot{\tilde{\mathbf{x}}}+\dot{\boldsymbol{\omega}}^{T}\left(\boldsymbol{\omega} \times \mathbf{I}_{\mathbf{e}} \boldsymbol{\omega}\right)+\frac{1}{2} \ddot{\boldsymbol{\beta}}^{T} \mathbf{I}_{\mathbf{m}} \ddot{\boldsymbol{\beta}}
$$

where:

$$
\left[\mathbf{M}_{\mathbf{e}}\right]=\left[\begin{array}{cc}
m_{e} \mathbf{I}_{\mathbf{3}} & \mathbf{0}_{\mathbf{3}} \\
\mathbf{0}_{\mathbf{3}} & \mathbf{I}_{\mathbf{e}}
\end{array}\right]
$$

where $\mathbf{I}_{\mathbf{e}}$ denotes the inertia tensor of the end-effector. The elements of the diagonal matrix, $\mathbf{I}_{\mathbf{m}}$, in Eq. (10) indicate the moment of inertia of the set, consisting of the rotor, gears, and the output shaft of the motors, which is obtained based on the output shaft rotation. The generalized power of the system is:

$$
\dot{W}=\boldsymbol{\tau}^{T} \dot{\boldsymbol{\beta}}
$$

where $\boldsymbol{\tau}$ is the torque on the motor output shaft. Substituting Eqs. (8)-(11) in the Gibbs-Appel formula, the following equations can be obtained:

$$
\begin{aligned}
& {\left[\mathbf{M}_{\mathbf{e}}\right] \ddot{\tilde{\mathbf{x}}}+\left[\begin{array}{c}
\mathbf{0}_{3 \times 1} \\
\boldsymbol{\omega} \times \mathbf{I}_{\mathbf{e}} \boldsymbol{\omega}
\end{array}\right]+\left[\begin{array}{lll}
\mathbf{0}_{\mathbf{1} \times \mathbf{2}} & -m_{e} g & \mathbf{0}_{\mathbf{1} \times \mathbf{3}}
\end{array}\right]^{\mathbf{T}}} \\
& -r_{p} \mathbf{A}_{\mathbf{1}}{ }^{\mathbf{T}}\left(\mathbf{K} \boldsymbol{\Delta} \mathbf{q}+\mathbf{C}_{\mathbf{d}} \boldsymbol{\Delta} \dot{\mathbf{q}}\right)=\mathbf{0}, \\
& \mathbf{I}_{\mathbf{m}} \ddot{\boldsymbol{\beta}}+\frac{r_{p}}{2} \operatorname{diag} g^{-1}\left(\mathbf{q}_{\mathbf{u}}\right) \operatorname{diag}\left(\mathbf{q}+\mathbf{q}_{\mathbf{u}}\right) \mathbf{K} \boldsymbol{\Delta} \mathbf{q} \\
& +r_{p} \mathbf{C}_{\mathbf{d}} \dot{\Delta} \mathbf{q}=\boldsymbol{\tau}
\end{aligned}
$$

where the symbol "diag (-)" indicates that the vector in the parenthesis is converted to a diagonal matrix. Since the changes of the cable length are small, the following equation can be considered:

$$
\operatorname{diag}^{-1}\left(\mathbf{q}_{\mathbf{u}}\right) \operatorname{diag}\left(\mathbf{q}+\mathbf{q}_{\mathbf{u}}\right) \approx 2 \mathbf{I}_{\mathbf{6}}
$$

Therefore, Eq. (13) can be simplified as:

$$
\mathbf{I}_{\mathbf{m}} \ddot{\boldsymbol{\beta}}+r_{p}\left(\mathbf{K} \boldsymbol{\Delta} \mathbf{q}+\mathbf{C}_{\mathbf{d}} \dot{\Delta} \mathbf{q}\right)=\boldsymbol{\tau}
$$

In order to express the rotational motion of the endeffector in terms of Euler angles, the angular velocity and acceleration in Eq. (12) are replaced with the equivalent equations in terms of Euler angle derivatives (see Eq. (A.1) in Appendix A). After multiplying the two sides of the equation by matrix $\mathbf{A}_{\mathbf{3}}^{\mathbf{T}}$, the dynamic equations can be shown in the following form:

$$
\mathbf{M} \ddot{\mathbf{z}}+\mathbf{C}+\mathbf{G}=\tau_{\text {tot. }},
$$

where:

$$
\mathbf{z}=\left[\begin{array}{c}
\mathbf{x} \\
\boldsymbol{\beta}
\end{array}\right]
$$

$\mathbf{M}, \mathbf{C}, \mathbf{G}$, and $\boldsymbol{\tau}_{\text {tot }}$ are defined in Box I.

\section{Stability analysis of the robot with elastic cables}

In [9], the asymptotic stability of the system is shown using a damping term in the controller, where the feedback of the tension rate is needed. However, the stability of the system can be achieved by approximation of the cables dissipation energy using Rayleigh viscous damping function. The dynamics of 


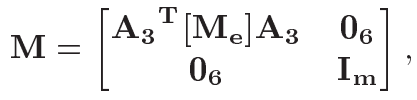

$$
\begin{aligned}
& \mathbf{C}=\left[\mathbf { A } _ { \mathbf { 3 } } ^ { \mathbf { T } } \left(\left[\mathbf{M}_{\mathbf{e}}\right] \mathbf{A}_{\mathbf{4}}+\left[\begin{array}{c}
\mathbf{0}_{3 \times 1} \\
\left.\left.\mathbf{P} \dot{\boldsymbol{\psi}} \times \mathbf{I}_{\mathbf{e}} \mathbf{P} \dot{\boldsymbol{\psi}}\right]-r_{p} \mathbf{A}_{\mathbf{1}}^{\mathbf{T}}\left(\mathbf{K} \boldsymbol{\Delta} \mathbf{q}+\mathbf{C}_{\mathbf{d}} \boldsymbol{\Delta} \dot{\mathbf{q}}\right)\right) \\
r_{p}\left(\mathbf{K} \mathbf{\Delta} \mathbf{q}+\mathbf{C}_{\mathbf{d}} \boldsymbol{\Delta} \dot{\mathbf{q}}\right)
\end{array}\right],\right.\right. \\
& \mathbf{G}=\left[\begin{array}{c}
\mathbf{0}_{2 \times 1} \\
-m_{e} g \\
\mathbf{0}_{9 \times 1}
\end{array}\right] \quad \boldsymbol{\tau}_{\text {tot. }}=\left[\begin{array}{c}
\mathbf{0}_{\mathbf{6} \times \mathbf{1}} \\
\boldsymbol{\tau}
\end{array}\right] \text {. }
\end{aligned}
$$

the flexible system can be investigated by dividing the dynamic equations into the slow and fast dynamics using singular perturbation techniques [23,24]. In order to express the flexible system equations in the form of singular perturbations, the state $u$ and the small parameter $\varepsilon$ are defined:

$$
\begin{aligned}
& \mathbf{u}=E A \boldsymbol{\Delta} \mathbf{q}, \\
& \varepsilon^{2}=\frac{1}{E A}, \quad c_{d}=\frac{\alpha}{\varepsilon}, \quad \alpha=O(1) .
\end{aligned}
$$

The order considered for the damping coefficient, $c_{d}$, is selected based on the analogy with a mass-springdamper system, where the damping coefficient is proportional to the square root of the spring constant. Using Eqs. (17) and (18), the angular acceleration vector is obtained as:

$$
\ddot{\boldsymbol{\beta}}=\frac{\varepsilon^{2}}{r_{p}} \ddot{\mathbf{u}}+\mathbf{A}_{\mathbf{1}} \mathbf{A}_{\mathbf{3}} \ddot{\mathbf{x}}+\mathbf{A}_{\mathbf{1}} \mathbf{A}_{\mathbf{4}}+\mathbf{A}_{\mathbf{2}} \text {. }
$$

Considering Eqs. (17)-(19), the dynamic equation (16) can be rewritten as:

$$
\begin{aligned}
& \mathbf{A}_{\mathbf{3}}^{\mathbf{T}}\left(\left[\mathbf{M}_{\mathbf{e}}\right]+\mathbf{A}_{\mathbf{1}}{ }^{T} \mathbf{I}_{\mathbf{m}} \mathbf{A}_{\mathbf{1}}\right) \mathbf{A}_{\mathbf{3}} \ddot{\mathbf{x}}+\mathbf{A}_{\mathbf{3}} \mathbf{T}\left(\left[\mathbf{M}_{\mathbf{e}}\right] \mathbf{A}_{\mathbf{4}}\right. \\
& +\left[\begin{array}{c}
\mathbf{0}_{3 \times 1} \\
\left.\mathbf{P} \dot{\boldsymbol{\psi}} \times \mathbf{I}_{\mathbf{e}} \mathbf{P} \dot{\boldsymbol{\psi}}\right]
\end{array}\right]+\mathbf{A}_{\mathbf{1}}{ }^{T} \mathbf{I}_{\mathbf{m}}\left(\mathbf{A}_{\mathbf{1}} \mathbf{A}_{\mathbf{4}}+\mathbf{A}_{\mathbf{2}}\right) \\
& \left.+\left[\begin{array}{c}
\mathbf{0}_{2 \times 1} \\
-m_{e} g \\
\mathbf{0}_{3 \times 1}
\end{array}\right]\right)=\mathbf{A}_{\mathbf{3}}{ }^{\mathbf{T}} \mathbf{A}_{\mathbf{1}}{ }^{T}\left(\boldsymbol{\tau}-\frac{\varepsilon^{2} \mathbf{I}_{\mathbf{m}}}{r_{p} \alpha} \ddot{\mathbf{u}}\right), \\
& \mathbf{I}_{\mathbf{m}}\left(\frac{\varepsilon^{2}}{r_{p}} \ddot{\mathbf{u}}+\mathbf{A}_{\mathbf{1}} \mathbf{A}_{\mathbf{3}} \ddot{\mathbf{x}}+\mathbf{A}_{1} \mathbf{A}_{\mathbf{4}}+\mathbf{A}_{\mathbf{2}}\right) \\
& \quad+r_{p} \operatorname{diag}^{-\mathbf{1}}\left(\mathbf{q}_{\mathbf{u}}\right)\left(\mathbf{u}+c_{d} \varepsilon^{2} \dot{\mathbf{u}}\right)=\boldsymbol{\tau} .
\end{aligned}
$$

In the above equations, $\mathbf{x}$ is the slow dynamic state and $\mathbf{u}$ represents the fast dynamic state produced by the flexibility of the system. The reduced model of the equations can be obtained by considering $\varepsilon=0$ and $\mathbf{q}=\mathbf{q}_{\mathbf{u}}$. According to Tikhonov theory [24], the states $\mathbf{x}$ and $\mathbf{u}$ can be approximated as follows:

$$
\left\{\begin{array}{l}
\mathbf{x}=\mathbf{x}_{\mathbf{0}}(t)+\mathbf{O}(\varepsilon) \\
\mathbf{u}=\mathbf{u}_{\mathbf{0}}(t)+\boldsymbol{\delta}(\tau)+\mathbf{O}(\varepsilon)
\end{array}\right.
$$

where $\tau=\frac{t}{\varepsilon}$ and $\delta$ are the time scale and the variable corresponding to the fast dynamic of the system. Ignoring the higher order terms, the first and second derivatives of $\mathbf{u}$ are:

$$
\left\{\begin{array}{l}
\dot{\mathbf{u}}=\dot{\mathbf{u}}_{\mathbf{0}}+\frac{1}{\varepsilon} \frac{\mathrm{d} \delta}{\mathrm{d} \tau} \\
\ddot{\mathbf{u}}=\ddot{\mathbf{u}}_{\mathbf{O}}+\frac{1}{\varepsilon^{2}} \frac{\mathrm{d}^{2} \delta}{\mathrm{d} \tau^{2}}
\end{array}\right.
$$

Ignoring the order $\varepsilon^{2}$ and the fast dynamic, Eq. (21) can be rewritten as:

$$
\begin{aligned}
\mathbf{u}_{\mathbf{0}} & +\alpha \varepsilon \dot{\mathbf{u}}_{0}=\frac{\operatorname{diag}\left(\mathbf{q}_{\mathbf{u}}\right)}{r_{p}}\left(\boldsymbol{\tau}_{\mathbf{0}}-\mathbf{I}_{\mathbf{m}}\left(\mathbf{A}_{\mathbf{1}_{\mathbf{o}}} \mathbf{A}_{\mathbf{3}_{\mathbf{0}}} \ddot{\mathbf{x}}_{\mathbf{0}}\right.\right. \\
& \left.\left.+\mathbf{A}_{\mathbf{1}_{\mathbf{o}}} \mathbf{A}_{\mathbf{4}_{\mathbf{0}}}+\mathbf{A}_{\mathbf{2}_{\mathbf{o}}}\right)\right)
\end{aligned}
$$

where $\tau_{0}$ is the torque obtained from the reduced model and $\tau_{\delta}$ denotes the torque considered for damping the vibrations of the fast dynamic. Considering $\tau=\tau_{\mathbf{0}}+\tau_{\delta}$ and substituting Eq. (24) in Eq. (21), the boundary layer equation is obtained as:

$$
\frac{\mathbf{I}_{\mathbf{m}}}{r_{p}} \frac{\mathrm{d}^{2} \boldsymbol{\delta}}{\mathrm{d} \tau^{2}}+r_{p} \operatorname{diag}^{-1}\left(\mathbf{q}_{\mathbf{u}}\right)\left(\boldsymbol{\delta}+\alpha \frac{\mathrm{d} \boldsymbol{\delta}}{\mathrm{d} \tau}\right)=\boldsymbol{\tau}_{\boldsymbol{\delta}}
$$

If the fast dynamic term of the controller is considered to be $\tau_{\delta}=-\mathbf{K}_{\delta} \varepsilon \dot{\mathbf{u}}$, Eq. (25) can be written as:

$$
\begin{aligned}
{\left[\begin{array}{l}
\dot{\boldsymbol{\delta}} \\
\ddot{\delta}
\end{array}\right] } & =\left[\begin{array}{cc}
\mathbf{0} & \mathbf{1} \\
-\mathbf{K}_{\mathbf{1}} & -\mathbf{K}_{\mathbf{2}}
\end{array}\right]\left[\begin{array}{l}
\boldsymbol{\delta} \\
\dot{\boldsymbol{\delta}}
\end{array}\right], \\
\mathbf{K}_{\mathbf{1}} & =\frac{r_{p}{ }^{2} E A}{\mathbf{I}_{\mathbf{m}} \operatorname{diag}\left(\mathbf{q}_{\mathbf{u}}\right)},
\end{aligned}
$$




$$
\mathbf{K}_{\mathbf{2}}=\frac{r_{p}{ }^{2} \sqrt{E A}}{\mathbf{I}_{\mathbf{m}}}\left(\frac{\mathbf{K}_{\delta}}{r_{p}}+\frac{\alpha}{\operatorname{diag}\left(\mathbf{q}_{\mathbf{u}}\right)}\right)
$$

where $\mathbf{K}_{\boldsymbol{\delta}}$ is a positive semi-definite matrix. Considering the control torque obtained for the rigid model and the kinematic equations, the dynamic equation (20) becomes:

$$
\begin{aligned}
& {\left[\begin{array}{c}
\dot{\mathbf{e}} \\
\ddot{\mathbf{e}}
\end{array}\right]=\left[\begin{array}{cc}
\mathbf{0} & \mathbf{I} \\
-\mathbf{K}_{\mathbf{p}} & -\mathbf{K}_{\mathbf{d}}
\end{array}\right]\left[\begin{array}{c}
\mathbf{e} \\
\dot{\mathbf{e}}
\end{array}\right]+\left[\begin{array}{cc}
\mathbf{0} & \mathbf{0} \\
\mathbf{C}_{\delta_{\mathbf{p}}} & \mathbf{C}_{\delta_{\mathbf{d}}}
\end{array}\right]\left[\begin{array}{c}
\delta \\
\dot{\delta}
\end{array}\right]} \\
& \mathbf{C}_{\delta_{\mathbf{p}}}=r_{p} \mathbf{M}^{-1}\left(\mathbf{A}_{\mathbf{1}} \mathbf{A}_{\mathbf{3}}\right)^{\mathbf{T}} \operatorname{diag}^{-1}\left(\mathbf{q}_{\mathbf{u}}\right) \\
& \mathbf{C}_{\delta_{\mathbf{d}}}=c_{d} \mathbf{C}_{\delta_{\mathbf{p}}}
\end{aligned}
$$

The stability analysis is performed with the Lyapunov candidate as:

$$
\begin{aligned}
& V=\frac{1}{2}\left(\dot{\mathbf{e}}^{\mathbf{T}} \dot{\mathbf{e}}+\mathbf{e}^{\mathbf{T}} \mathbf{K}_{\mathbf{p}} \mathbf{e}+\left[\begin{array}{ll}
\delta^{\mathbf{T}} & \dot{\delta}^{\mathbf{T}}
\end{array}\right]\right. \\
& \left.\left[\begin{array}{cc}
r_{p}^{2}(E A+\sqrt{E A} \alpha) \mathbf{I} & \mathbf{I}_{\mathbf{m}} \operatorname{diag}\left(\mathbf{q}_{\mathbf{u}}\right) \\
\mathbf{I}_{\mathbf{m}} \operatorname{diag}\left(\mathbf{q}_{\mathbf{u}}\right) & \mathbf{I}_{\mathbf{m}} \operatorname{diag}\left(\mathbf{q}_{\mathbf{u}}\right)
\end{array}\right]\left[\begin{array}{c}
\delta \\
\dot{\delta}
\end{array}\right]\right)
\end{aligned}
$$

The derivative of Eq. (28) is obtained as:

$$
\begin{aligned}
\dot{V}= & -\dot{\mathbf{e}}^{\mathbf{T}} \mathbf{K}_{\mathbf{d}} \dot{\mathbf{e}}+\dot{\mathbf{e}}^{\mathbf{T}}\left[\begin{array}{ll}
\mathbf{C}_{\delta_{\mathbf{p}}} & \mathbf{C}_{\boldsymbol{\delta}_{\mathbf{d}}}
\end{array}\right]\left[\begin{array}{l}
\boldsymbol{\delta} \\
\dot{\boldsymbol{\delta}}
\end{array}\right]-\left[\begin{array}{ll}
\boldsymbol{\delta}^{\mathbf{T}} & \dot{\boldsymbol{\delta}}^{\mathbf{T}}
\end{array}\right] \\
& {\left[\begin{array}{cc}
r_{p}{ }^{2} E A \mathbf{I} & \mathbf{0} \\
\mathbf{0} & \alpha r_{p}{ }^{2} \sqrt{E A} \mathbf{I}
\end{array}\right]\left[\begin{array}{l}
\boldsymbol{\delta} \\
\dot{\boldsymbol{\delta}}
\end{array}\right] }
\end{aligned}
$$

The cable stiffness is considered to be constant during the derivative operations. If a condition is found that makes $\dot{V}$ negative semi-definite, then the stability of the origin is proven. The upper bound of the terms in Eq. (29) are considered as:

$$
\begin{aligned}
& -\dot{\mathbf{e}}^{\mathbf{T}} \mathbf{K}_{\mathbf{d}} \dot{\mathbf{e}} \leq-\lambda_{\min }\left(\mathbf{K}_{\mathbf{d}}\right)\|\dot{\mathbf{e}}\|=-\lambda_{m e}\|\dot{\mathbf{e}}\|^{2}, \\
& \dot{\mathrm{e}}^{\mathrm{T}}\left[\begin{array}{ll}
\mathbf{C}_{\delta_{\mathrm{p}}} & \mathbf{C}_{\delta_{\mathrm{d}}}
\end{array}\right]\left[\begin{array}{c}
\delta \\
\dot{\delta}
\end{array}\right] \leq\|\dot{\mathrm{e}}\|\left\|\left[\begin{array}{c}
\delta \\
\dot{\delta}
\end{array}\right]\right\| \sigma_{\max }\left(\left[\begin{array}{ll}
\mathbf{C}_{\delta_{\mathrm{p}}} & \mathbf{C}_{\delta_{\mathrm{d}}}
\end{array}\right]\right) \\
& =\sigma_{m}\|\dot{\mathbf{e}}\|\left\|\left[\begin{array}{c}
\delta \\
\dot{\delta}
\end{array}\right]\right\| \\
& -\left[\begin{array}{c}
\boldsymbol{\delta} \\
\dot{\boldsymbol{\delta}}
\end{array}\right]^{\mathbf{T}}\left[\begin{array}{cc}
r_{p}{ }^{2} E A \mathbf{I} & \mathbf{0} \\
\mathbf{0} & \alpha r_{p}{ }^{2} \sqrt{E A} \mathbf{I}
\end{array}\right]\left[\begin{array}{c}
\boldsymbol{\delta} \\
\dot{\boldsymbol{\delta}}
\end{array}\right] \leq \\
& -\lambda_{\min }\left(\left[\begin{array}{cc}
r_{p}^{2} E A \mathbf{I} & \mathbf{0} \\
\mathbf{0} & \alpha r_{p}^{2} \sqrt{E A} \mathbf{I}
\end{array}\right]\right)\left\|\left[\begin{array}{c}
\boldsymbol{\delta} \\
\dot{\boldsymbol{\delta}}
\end{array}\right]\right\| \\
& =-\lambda_{m} \delta\left\|\left[\begin{array}{c}
\delta \\
\dot{\delta}
\end{array}\right]\right\|^{2}
\end{aligned}
$$

As a result, the upper limit of $\dot{V}$ is:

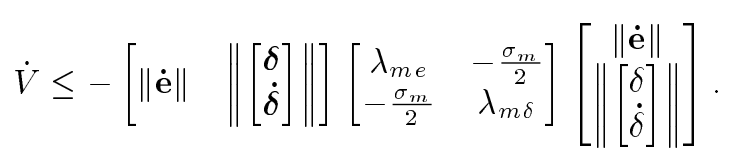

In order to make $\dot{V} \leq 0$, the following condition should be satisfied:

$$
\lambda_{m e} \lambda_{m \delta} \geq \frac{\sigma_{m}^{2}}{4} .
$$

Therefore, considering the cable damping and the suitable choice of derivative gain in the rigid model based controller, the stability of the system can be guaranteed.

\section{Control approaches}

As explained in the previous section, the torque $\tau$ can be obtained using a composite control, i.e. $\boldsymbol{\tau}=\boldsymbol{\tau}_{0}+\boldsymbol{\tau}_{\delta}$, where $\boldsymbol{\tau}_{\delta}$ is obtained using the time rate of the cable tension. If instead of the time derivative of the cable tension, a direct feedback from the endeffector is present, the following equivalent value of $\tau_{\delta}$ can be used:

$$
\boldsymbol{\tau}_{\delta}=-\mathbf{K}_{\delta} r_{p}\left(\dot{\boldsymbol{\beta}}-\mathbf{A}_{\mathbf{1}} \mathbf{A}_{\mathbf{3}} \dot{\mathbf{x}}\right)
$$

If the cables have no damping properties, ignoring the term $\tau_{\delta}$ in the controller results in increase in the cable tension oscillation and, consequently, the end-effector vibration. Therefore, in order to suppress the vibration using the composite control method, the feedback from the cable tension rate or the pose and velocities of the end-effector are needed. The desired error dynamic of the flexible system can also be achieved using the feedback linearization method and the feedback only from the end-effector. If no damping is assumed in the cables, the first 6 rows in Eq. (16) can be rewritten in the following compact form:

$$
\tilde{\mathbf{M}} \ddot{\mathbf{x}}+\tilde{\mathbf{C}}+\tilde{\mathbf{G}}=\Delta \mathbf{q}
$$

According to the last 6 rows of Eq. (16), the 2nd derivative of $\boldsymbol{\beta}$ has a direct relation with $\boldsymbol{\tau}$. Following the steps in Appendix B, the control torque:

$$
\begin{aligned}
\boldsymbol{\tau}= & \frac{\mathbf{I}_{\mathbf{m}}}{r_{p}}\left(\ddot{\tilde{\mathbf{M}}} \mathbf{x}^{(2)}+2 \dot{\tilde{\mathbf{M}}} \mathbf{x}^{(3)}+\tilde{\mathbf{M}} \boldsymbol{v}+\ddot{\tilde{\mathbf{C}}}+\ddot{\tilde{\mathbf{G}}}-\ddot{\mathbf{q}}\right) \\
& +r_{p} \mathbf{K}(\tilde{\mathbf{M}} \ddot{\mathbf{x}}+\tilde{\mathbf{C}}+\tilde{\mathbf{G}})
\end{aligned}
$$

linearizes the error dynamic. In Eq. (37) $\boldsymbol{\nu}=\overline{\mathbf{x}}_{\mathbf{d}}^{(4)}+$ $\sum_{i=0}^{3} k_{i} \mathbf{e}^{(i)}$ and $\mathbf{e}=\mathbf{x}_{\mathbf{d}}-\mathbf{x}$. Also, $\mathbf{x}^{(\mathbf{i})}$ denotes the $i$ th derivative of $\mathbf{x}$. The coefficients $k_{i}$ can be obtained using the desired error dynamic. It is notable that the 
states needed to be fed back to the controller can be any of the following equivalent sets:

$$
(\mathrm{x}, \dot{\mathrm{x}}, \ddot{\mathrm{x}}, \dddot{\mathrm{x}}),(\mathrm{x}, \dot{\mathrm{x}}, \boldsymbol{\beta}, \dot{\boldsymbol{\beta}}),(\mathrm{x}, \dot{\mathrm{x}}, \mathbf{T}, \dot{\mathrm{T}}),
$$

where $\mathbf{T}=\mathbf{K} \boldsymbol{\Delta} \mathbf{q}$. The matrices $\tilde{\mathbf{M}}, \tilde{\mathbf{C}}$, and $\tilde{\mathbf{G}}$ in Eq. (36) are obtained by multiplying the two sides of the first six rows of Eq. (13) by $\left(r_{p} \mathbf{A}_{1}^{T} \mathbf{K}\right)^{-1}$. Since the matrix $\mathbf{A}_{1}$ includes the Jacobian matrix $\boldsymbol{\Lambda}^{\mathbf{T}}$, obtaining the derivatives of $\tilde{\mathbf{M}}, \tilde{\mathbf{C}}$, and $\tilde{\mathbf{G}}$ in Eq. (37), which include matrix $\left(\mathbf{A}_{1}^{\mathbf{T}}\right)^{-1}$, needs substantial computations. In addition, providing the necessary feedback of the states as in Eq. (38) needs additional sensors compared with the rigid model control, which increases the complication for obtaining the control effort. In composite control approach, the slow dynamic term $\tau_{\mathbf{0}}$ is obtained using the feedback from encoders of motors, while calculating the fast dynamic term $\tau_{\delta}$ requires the feedback from the end-effector pose, and velocities or the cable tension rate. In this paper, another approach for simplifying the controller computations as well as avoiding the use of additional feedback is considered. Considering that the dynamics of the flexible system converge to the rigid model dynamics, the control torque is calculated using a rigid model based control approach and the feedback from the actuators. In addition, the end-effector vibration is suppressed using a robust input shaping method. The control torque based on the rigid model is obtained employing the Feedback Linearization method as follows:

$$
\begin{aligned}
& \tau=\overline{\mathbf{F}}^{-1}\left(\overline{\mathbf{M}} v_{\mathrm{FL}}+\overline{\mathbf{C}}+\overline{\mathbf{G}}\right), \\
& v_{\mathrm{FL}}=\ddot{\mathrm{x}}_{\mathbf{d}}+\mathbf{K}_{\mathrm{p}} \mathbf{e}+\mathbf{K}_{\mathrm{d}} \dot{\mathbf{e}},
\end{aligned}
$$

where:

$$
\mathbf{e}=\mathbf{x}_{\mathbf{d}}-\mathbf{x}, \quad \dot{\mathbf{e}}=\dot{\mathbf{x}}_{\mathbf{d}}-\dot{\mathbf{x}} .
$$

The inertia matrix $\overline{\mathbf{M}}$, centrifugal and Coriolis matrix $\overline{\mathbf{C}}$, gravity matrix $\overline{\mathbf{G}}$, and the torque coefficient matrix $\overline{\mathbf{F}}$ are obtained using the rigid model of the system. Similar to Eq. (39), a typical sliding mode controller can be obtained by a small modification in the control input $\boldsymbol{v}_{F L}$ as follows:

$$
\begin{aligned}
& v_{\mathrm{SMC}}=\ddot{\mathbf{x}}_{\mathbf{d}}+\mathbf{K}_{\mathrm{d}} \dot{\mathbf{e}}+\mathbf{K} \operatorname{sign}(\mathbf{s}), \\
& \mathbf{s}=\dot{\mathbf{e}}+\mathbf{K}_{\mathrm{d}} \mathbf{e} .
\end{aligned}
$$

Matrix $\mathbf{K}$ is strictly positive definite. In spite of some bounded disturbances or dynamic uncertainties, the discontinuous function included in Eq. (40) can guarantee that the trajectories of the system reach the invariant set $\mathbf{s}=\mathbf{0}$ in a finite time. On the sliding surface, the error dynamics is defined by the equation of the set. In order to avoid steady state errors, integral action can be considered in the controller. To this aim, the sliding surface can be defined as:

$$
\mathbf{s}=\dot{\mathbf{e}}+\mathbf{K}_{\mathbf{d}} \mathbf{e}+\mathbf{K}_{\mathbf{p}} \int_{0}^{t} \mathbf{e} d \tau .
$$

By modifying Eq. (40) to:

$$
v_{\mathrm{ISMC}}=\ddot{\mathrm{x}}_{\mathrm{d}}+\mathbf{K}_{\mathrm{d}} \dot{\mathbf{e}}+\mathbf{K}_{\mathrm{p}} \mathbf{e}+\mathbf{K} \operatorname{sign}(\mathbf{s}),
$$

the error dynamics of the integral sliding mode controller on the sliding surface become identical to those of the FL controller:

$$
\ddot{\mathbf{e}}+\mathbf{K}_{\mathrm{d}} \dot{\mathbf{e}}+\mathbf{K}_{\mathrm{p}} \mathbf{e}=\mathbf{0} .
$$

At the expense of chattering on the control input, the switching function ensures reaching the sliding surface in a finite time and remaining on the surface in the presence of uncertainties and disturbances with known bound. Thus far, different methods have been proposed to smooth the discontinuous control law by finding a trade-off between the control bandwidth and the tracking accuracy. Since this paper does not intend to address the uncertainty issues of the rigid model, the control law presented in Eq. (39) is utilized. The feedback from the end-effector in the Cartesian space is approximated using the direct kinematic equation and the data obtained from the encoders:

$$
\dot{\mathbf{x}}=\left(\begin{array}{ll}
\mathbf{A}_{1} & \mathbf{A}_{3}
\end{array}\right)^{-1} \dot{\boldsymbol{\beta}} .
$$

\section{Vibration reduction using input shaping}

Input shaping technique is based on convolving a desired input with a sequence of impulses. The amplitudes and the times of applied impulses are determined in such a way that at the end of command, the vibration caused by exciting the system natural modes is reduced or cancelled completely. If a good approximation of the natural frequency and damping exists, then the ratio of the vibration amplitude produced by a sequence of impulses to the vibration amplitude caused by a unit impulse is [25]:

$$
V(\omega, \zeta)=e^{-\zeta \omega t_{m}} \sqrt{C(\omega, \zeta)^{2}+S(\omega, \zeta)^{2}},
$$

where:

$$
\begin{aligned}
& C(\omega, \zeta)=\sum_{i=1}^{m} A_{i} e^{\zeta \omega t_{i}} \cos \left(\omega_{d} t_{i}\right), \\
& S(\omega, \zeta)=\sum_{i=1}^{m} A_{i} e^{\zeta \omega t_{i}} \sin \left(\omega_{d} t_{i}\right), \\
& \omega_{d}=\omega \sqrt{1-\zeta^{2}}
\end{aligned}
$$


$A_{i}$ and $t_{i}$ denote the amplitude and the time location of the $i$-th impulse, respectively, $\omega$ is natural frequency, $\zeta$ is damping, and $m$ represents the number of impulses. The main goal of the input shaping methods is reduction in the vibration amplitude ratio, $V$, using the formulations for the amplitude and time location of the impulses. A vibrational system may have more than one natural mode. If $m$ impulses are considered for a system with $n$ natural modes, then the shaped impulse sequence for the $p$-th mode can be expressed by:

$$
L_{p}=\sum_{i=1}^{m} A_{p, i} \delta\left(t-t_{p, i}\right)
$$

where:

$$
p=1 \ldots n \quad, \quad t_{p, i}=(i-1) \frac{\pi}{\omega_{p} \sqrt{1-\zeta_{p}^{2}}},
$$

where $\delta\left(t-t_{p, i}\right)$ denotes the impulse applied at instant $t_{p, i}$. The composite shaped impulse sequence $L_{m u l t}$ for a vibrational system with $n$ modes can be formed by convolving the sequences as:

$$
L_{m u l t}=L_{1} * \ldots * L_{n}
$$

where $*$ is the convolution operator. An impulse sequence works as a filter of the system input. Therefore, it causes a time delay equivalent to one cycle of the impulse sequence. When the designed composite sequence $L_{\text {mult }}$ is combined with any desired input, $r(t)$, a shaped input, $x(t)$, with a property that does not excite the natural modes is obtained as:

$$
x(t)=r(t) * L_{m u l t} .
$$

In practice, modeling error always exists. Considering a linear behavior for a nonlinear system to estimate the damping and natural frequency is one source of the errors. In order to robustify the input shaping method against the damping and natural frequency uncertainties, the constraint of making vibration amplitude ratio, $V$, equal to zero is relaxed to a specified tolerable level of vibration, $V_{\mathrm{tol}}$. One of the input shaping methods that benefits from this idea is EI shaper. For $V_{\mathrm{tol}}=5 \%$, the allowable band of frequency uncertainty of single hump EI shaper is $\pm 20 \%$. If more robustness is required, multi-hump EI shapers can be designed [26]. In this paper, vibration reduction is accomplished using undamped three-hump EI shaper, where the amplitudes and time locations of the impulses are:

$$
\left[\begin{array}{c}
A_{i} \\
t_{i}
\end{array}\right]=\left[\begin{array}{ccccc}
A_{1} & A_{2} & 1-2\left(A_{1}+A_{2}\right) & A_{2} & A_{1} \\
0 & 0.5 T & T & 1.5 T & 2 T
\end{array}\right]
$$

where:

$$
\begin{aligned}
& A_{1}=\frac{1+3 V_{\mathrm{tol}}+2 \sqrt{2 V_{\mathrm{tol}}\left(V_{\mathrm{tol}}+1\right)}}{16} ; \\
& A_{2}=\frac{1-V_{\mathrm{tol}}}{4} ; \\
& T=\frac{2 \pi}{\omega} .
\end{aligned}
$$

In the three-hump EI shaper, the allowable band of frequency uncertainty for $V_{\mathrm{tol}}=5 \%$ is nearly $\pm 50 \%$.

\section{Simulation}

In this section, several case studies are performed to show the effectiveness of the proposed approach. The first case study investigates convergence of the dynamic response of the flexible robot towards the rigid-cable robot. The next two case studies show the contributions of the proposed approach and the last compares the proposed controller with a composite controller. The coordinates of the cables endpoints on the platform $\mathbf{r}_{\mathbf{A}_{\mathbf{i}}}$ and on the end-effector $\mathbf{r}_{\mathbf{B}_{\mathbf{i}}}$ are

\begin{tabular}{|c|c|c|c|c|c|}
\hline Name & Symbol & & Value & & Unit \\
\hline Side length of the triangular upper plate of the platform & - & & 1.19 & & $\mathrm{~m}$ \\
\hline Side length of the triangular end-effector & - & & 0.17 & & $\mathrm{~m}$ \\
\hline Radius of the cable drums & $r_{p}$ & & 0.015 & & $\mathrm{~m}$ \\
\hline End-effector mass & $m_{e}$ & & 3.1 & & $\mathrm{~kg}$ \\
\hline Moment of inertia tensor of the end-effector & $I_{e}$ & {$\left[\begin{array}{c}0.0018 \\
0 \\
0\end{array}\right.$} & $\begin{array}{c}0 \\
0.0018 \\
0\end{array}$ & $\left.\begin{array}{c}0 \\
0 \\
0.0036\end{array}\right]$ & kg.m ${ }^{2}$ \\
\hline Equivalent moment of inertia of the motors & $\mathbf{I}_{\mathrm{m}}$ & & $0.03 \mathbf{I}_{6}$ & & $\mathrm{~kg} \cdot \mathrm{m}^{2}$ \\
\hline
\end{tabular}
obtained based on the side length of the triangular endeffector and the upper triangular plate of the platform as presented in Table 1 . This table also represents the inertia parameters as well as the drum radius of motors. The scheme for control gain selection is provided in Figure 2. Considering the error dynamics of the rigid

Table 1. Geometrical and inertia parameters. 


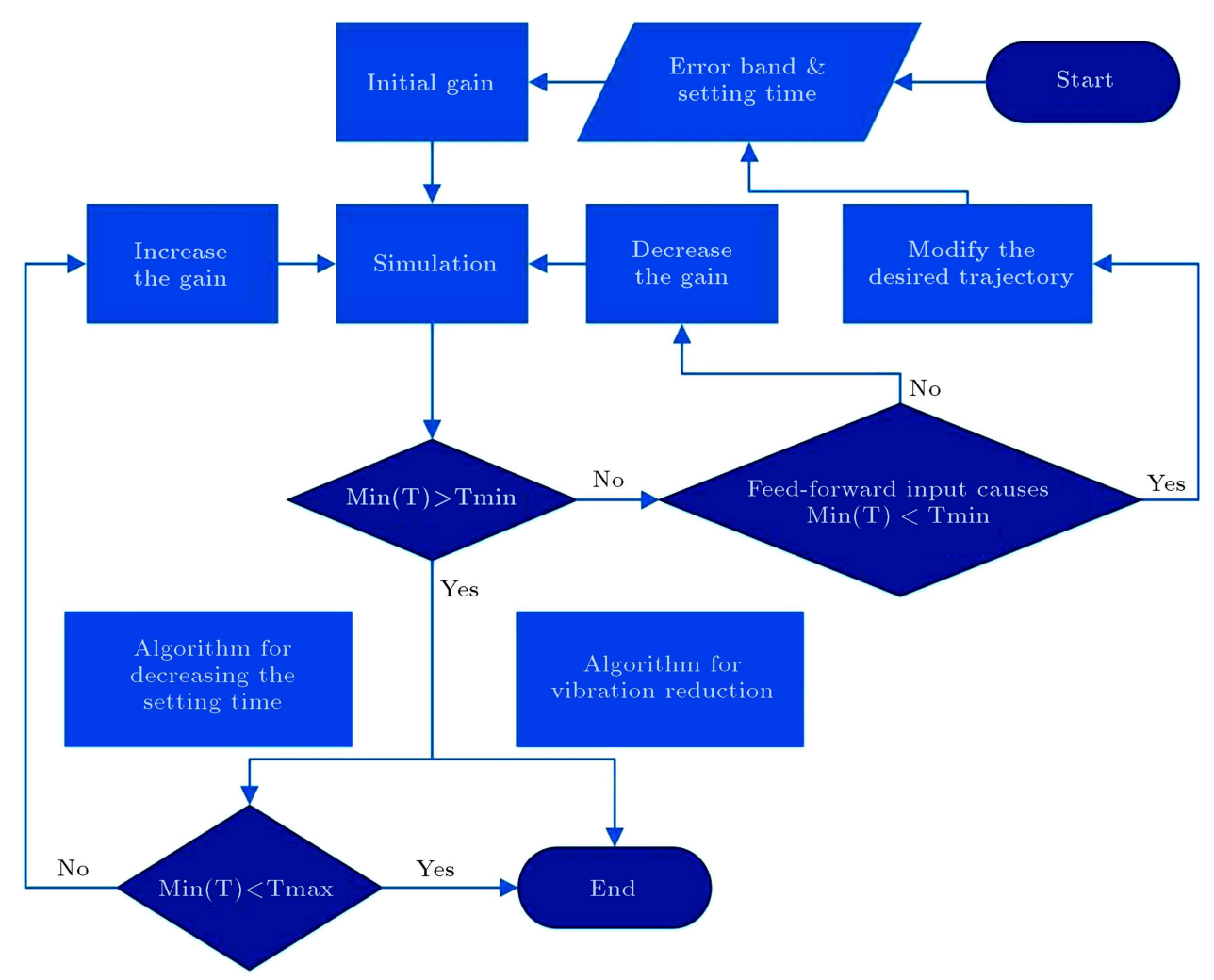

Figure 2. The scheme for control gain selection.

model and the initial errors, a primary guess about the control gain can be made using the given values of the positive settling band, $e_{s}$, and the settling time, $t_{s}$, as follows:

$$
e_{s}=\exp \left(-\frac{K_{d}}{2} t_{s}\right)\left|e_{0}\left(1+\frac{K_{d}}{2} t_{s}\right)+t_{s} \dot{e}_{0}\right|,
$$

where $e_{0}$ and $\dot{e}_{0}$ are the initial errors. In order to prevent the oscilation in the slow dynamic motion, Eq. (50) is obtained considering the following relation between the control gains:

$$
\mathbf{K}_{\mathbf{d}}=2 \sqrt{\mathbf{K}_{\mathbf{p}}} .
$$

Since the intended cable robot is of the underconstrained type, the cable tension cannot be arbitrarily increased. Large feedback control input or severe changes of the desired trajectory may lead to cable tension oscilation. This may result in cable slack. Considering the nonlinear dynamic of the flexible system, cable tension should be determined numerically. Therefore, in order to hold the positive tension condition, the control gains should be obtained by a trial and error algorithm. According to Figure 2, the cable tension should not become less than a given positive value, Tmin. In case of decrease in settling time, the minimum cable tension should fall within a given range of [Tmin, Tmax]. If in the simulation, the minimum cable tension becomes less than Tmin, it should be checked which part of the control input, i.e. feedforward or feedback, leads to the violation of the tension condition. To this end, a simulation of the rigid model with the trajectory obtained from the flexible model simulation and using the inverse dynamic is performed. Next, by checking the minimum tension condition, it can be decided whether the control gain should be decreased or the desired trajectory is not applicable and should be changed. If the minimum cable tension is above Tmin, the selected control gain can be used for the vibration reduction approach. However, in case of reducing the settling time, if the minimum cable tension exceeds Tmax, the control gain should be increased untill it falls within the given range.

It should be noted that the algorithm provided in Figure 2 assumes that the desired trajectory is smooth and the initial states of the system are not placed on the desired trajectory. If these assumptions are not satisfied and the tracking error is not in the considered band, the control gain is increased and the simulation is repeated. Moreover, when the initial states of the system are on the desired trajectory, the control gains are initially chosen with a blind guess and, then, the rest of the steps are followed.

\subsection{Dynamic response of the flexible robot}

As shown in Box II, the desired path considered for the first case study is circular such that the velocity of the end-effector equals zero in the beginning and end of the 


$$
\mathbf{x}_{\text {des }}=\left\{\begin{array}{l}
{\left[0.05 \cos \left(\frac{\pi t^{2}}{16}\right), 0.05 \sin \left(\frac{\pi t^{2}}{16}\right), 0.45,0,0,0\right]^{T} 0 \leq t<4} \\
{\left[0.05 \cos \left(\frac{\pi(8-t)^{2}}{16}\right),-0.05 \sin \left(\frac{\pi(8-t)^{2}}{16}\right), 0.45,0,0,0\right]^{T} ; 4 \leq t \leq 8}
\end{array}\right.
$$

Box II

path. The initial pose of the end-effector with respect to the desired path is:

$$
\mathbf{e}_{\mathbf{0}}=[-0.02,-0.02,0.3,0,0,0]^{T} .
$$

Control gain values are selected as:

$$
\left\{\begin{array}{l}
\mathbf{K}_{\mathbf{p}}=\operatorname{diag}(4.25,4.25,21.25,0.425,0.425,4.25) \\
\mathbf{K}_{\mathbf{d}}=2 \sqrt{\mathbf{K}_{\mathbf{p}}}
\end{array}\right.
$$

The control gains of the position are obtained considering the settling time and the error band in the rigid model as follows:

$$
\begin{aligned}
& \mathbf{t}_{\mathbf{s}}=[3,3,1.7]^{T}, \\
& \mathbf{e}_{\mathbf{s}}=[3,3,1]^{T} \mathrm{~mm} .
\end{aligned}
$$

The angular control gains are selected in such a way that during the tracking, the angular errors remain below 1 deg. Simulation of the closed loop system is performed for the rigid-cable robot as well as for the flexible robot using three stiffness values, i.e. $E A=$ $1.5 \mathrm{e} 3,1.5 \mathrm{e} 4$, and $1.5 \mathrm{e} 5$. Figure 3 shows the endeffector path in $X Y$ plane. Since the desired and initial values of Euler angles are set to zero, they show small variations. Therefore, frequency analysis of the data using FFT better reveals the dominant frequency of the system in the course of motion. As

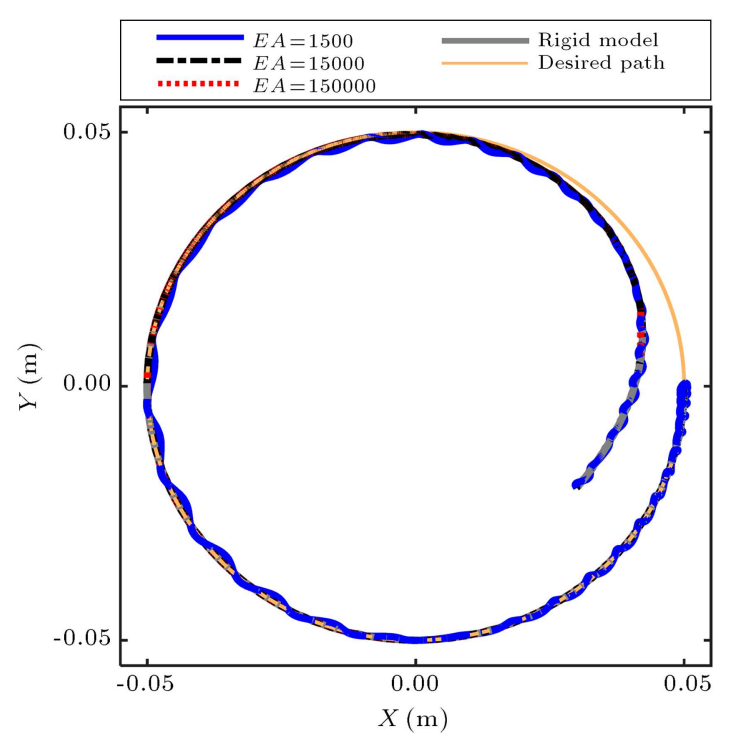

Figure 3. Circular path of the end-effector.

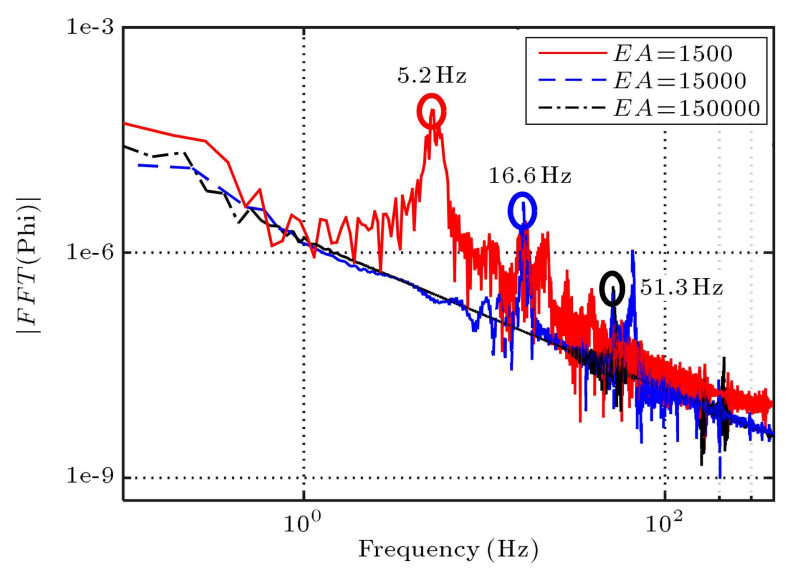

Figure 4. Frequency analysis of the 3rd Euler angle, $\varphi$.

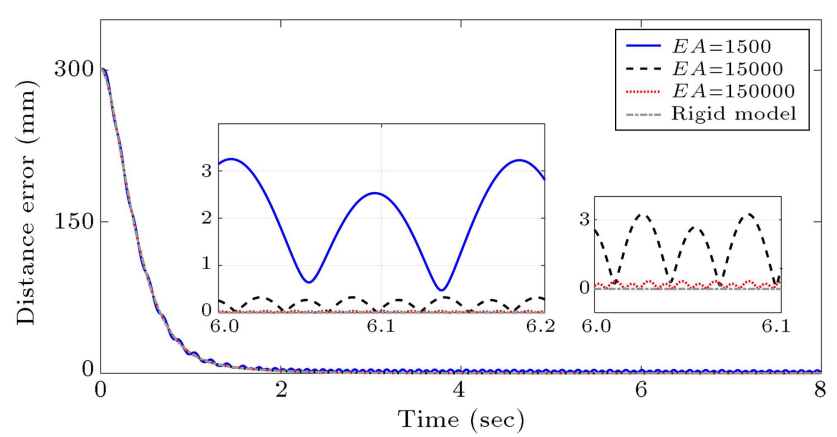

Figure 5. The distance between C.G. of the end-effector and the desired path.

shown in Figure 4, when $E A$ is multiplied by 10 , with a good approximate, the most dominant or the lowest natural frequency is multiplied by $\sqrt{10}$. In addition, an increase in the location of the lowest natural frequency decreases the amplitude vibration. Similarly, the distance between C.G. of the end-effector and the desired path in Figure 5 presents the same results. This is because with increase in $E A$, the frequency increases, the vibration amplitude decreases, and the system dynamic becomes closer to the rigidcable model.

The next two case studies in Sections 6.2 and 6.3 show the two contributions of this paper, i.e. improvement of settling time and vibration reduction. The last case study presented in Section 6.4 compares the proposed controller with a composite controller and investigates vibration reduction using an introduced index. Since a low cable flexibility is considered in Section 6.2 , vibration of the end-effector is not remarkable. 
However, using input shaping, cable tension oscillation is suppressed and, as a result, reaching the desired point can be achieved in a shorter time. The case study in Section 6.3 is performed with high cable flexibility. Therefore, the simulation is carried out for the purpose of reducing the vibration in the end-effector using three-hump EI input shaper. In the simulation, input shaping is applied to the desired input, which is expressed in the Cartesian space. Furthermore, the damping in the cables is ignored in order to show the performance of the proposed approach.

\subsection{Reducing the settling time}

In order to study the effectiveness of the proposed approach in the robot with low cable flexibility condition, a high stiffness of $E A=15 \mathrm{e} 5$ is selected. Two simulations are performed in this case study. In the first simulation, input shaping method is not applied. Using the first simulation result, the dominant natural frequencies of the system are obtained and used when applying the input shaping method in the second simulation. In these simulations, a control command for the end-effector to move from the initial point $\mathbf{x}_{\mathbf{0}}$ to the desired point $\mathbf{x}_{\mathbf{f}}$ is applied:

$$
\left\{\begin{array}{l}
\mathbf{x}_{\mathbf{0}}=\left[\begin{array}{llllll}
0 & 0 & 1.1 & 0 & 0 & 0
\end{array}\right]^{T} \\
\mathbf{x}_{\mathbf{f}}=\left[\begin{array}{llllll}
0.2 & 0.2 & 0.8 & 0 & 0 & \frac{\pi}{12}
\end{array}\right]^{T}
\end{array}\right.
$$

In order to calculate the initial control gains associated with the position and the third Euler angle of the end-effector, the settling time and the error band are considered as follows:

$$
\begin{aligned}
& \mathbf{t}_{\mathbf{s}}=[7.4,7.4,3.5,4.4]^{T}, \\
& \mathbf{e}_{\mathrm{s}}=[1 \mathrm{~mm}, 1 \mathrm{~mm}, 1 \mathrm{~mm}, 1 \mathrm{deg}]^{T} .
\end{aligned}
$$

The rest of the angular control gains are selected in such a way that during the tracking, the associated angular errors remain below $0.1 \mathrm{deg}$. Following the algorithm presented in Figure 2, the control gains which do not result in zero cable tension are obtained by:

$$
\left\{\begin{array}{l}
\mathbf{K}_{\mathbf{p}}=\operatorname{diag}(1,1,5,0.1,0.1,1) \\
\mathbf{K}_{\mathbf{d}}=2 \sqrt{\mathbf{K}_{\mathbf{p}}}
\end{array}\right.
$$

By considering the zero desired value for the first and the second Euler angles, the slow dynamic of these states becomes negligible. Therefore, frequency analysis of the states better reveals the vibration of the fast dynamic of the system. Figures 6 and 7 demonstrate the magnitude of FFT for these two Euler angles as well as cable tension, respectively. It is shown that the most dominant frequencies of the vibrational system are $32.7 \mathrm{~Hz}$ and $63.7 \mathrm{~Hz}$.

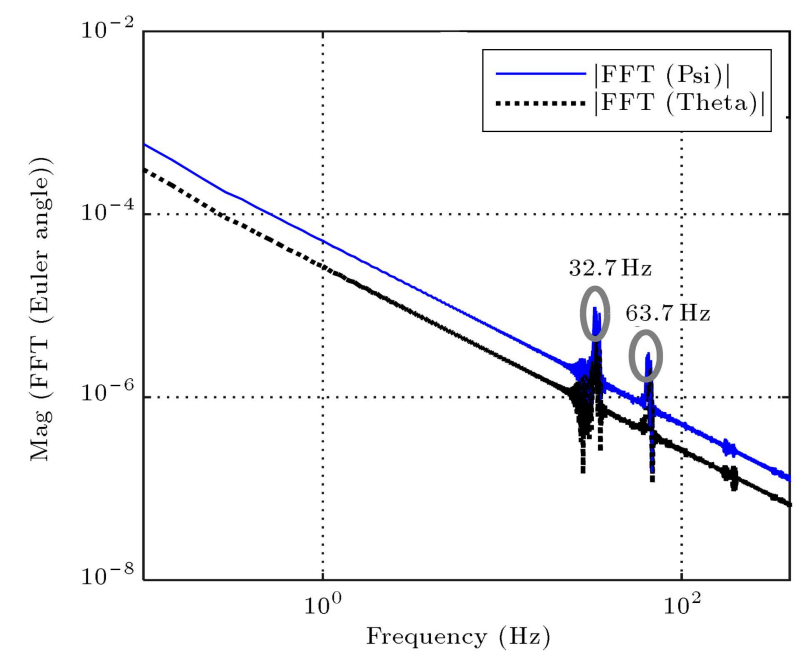

Figure 6. Frequency analysis of the 1 st and 2nd Euler angles.

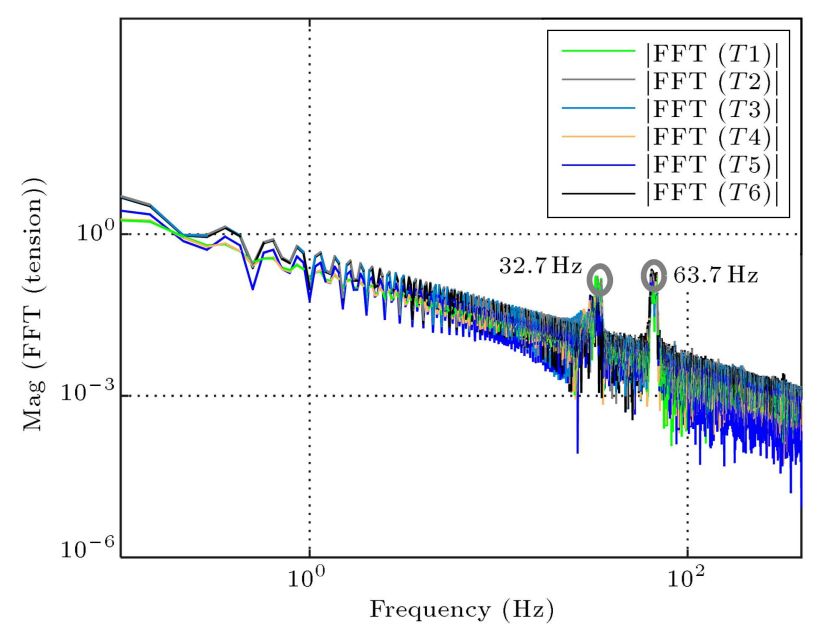

Figure 7. Frequency analysis of cables tension.

Since in cable modeling, damping is ignored, the parameter $\zeta$ is set to zero. The tolerable level of the vibration is selected to be $V_{\text {tol }}=5 \%$. Considering the natural frequency obtained in the previous simulation, as well as the tolerable level of the vibration amplitude ratio, the second simulation with three-hump EI input shaping is performed. Using the input shaping method, the oscillation amplitude of the cable tension with the previous control gains is reduced. Therefore, the control gain, $\mathbf{K}_{\mathbf{p}}$, for the second simulation is increased by 2.5 times the previous values to achieve almost the same minimum tension as that in the previous simulation:

$$
\left\{\begin{array}{l}
\mathbf{K}_{\mathbf{p}}=\operatorname{diag}(2.5,2.5,12.5,0.25,0.25,2.5) \\
\mathbf{K}_{\mathbf{d}}=2 \sqrt{\mathbf{K}_{\mathbf{p}}}
\end{array}\right.
$$

Figures 8 and 9 show that cable tension is significantly reduced after implementing the input shaping method compared with the simulation without input shaping. 


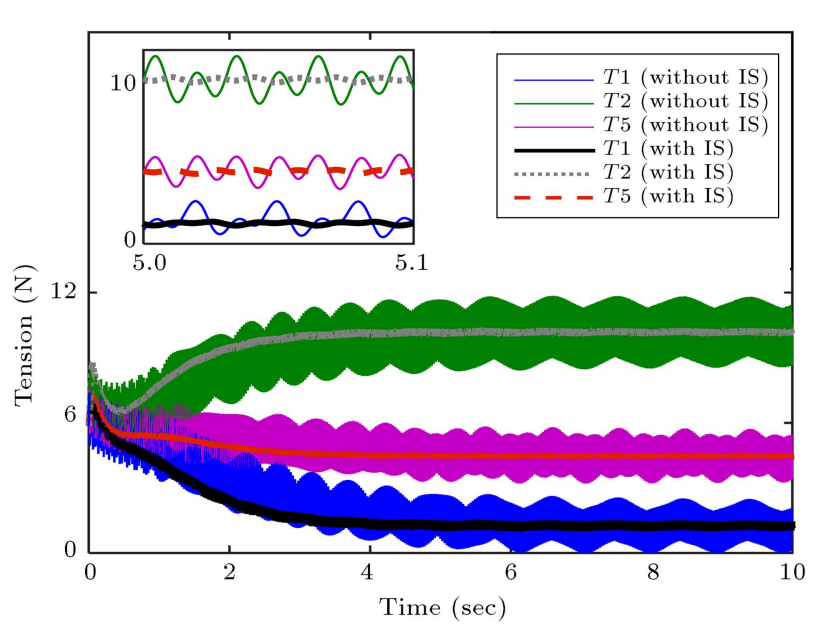

Figure 8. Tension of the 1st, 2nd, and 5th cables.

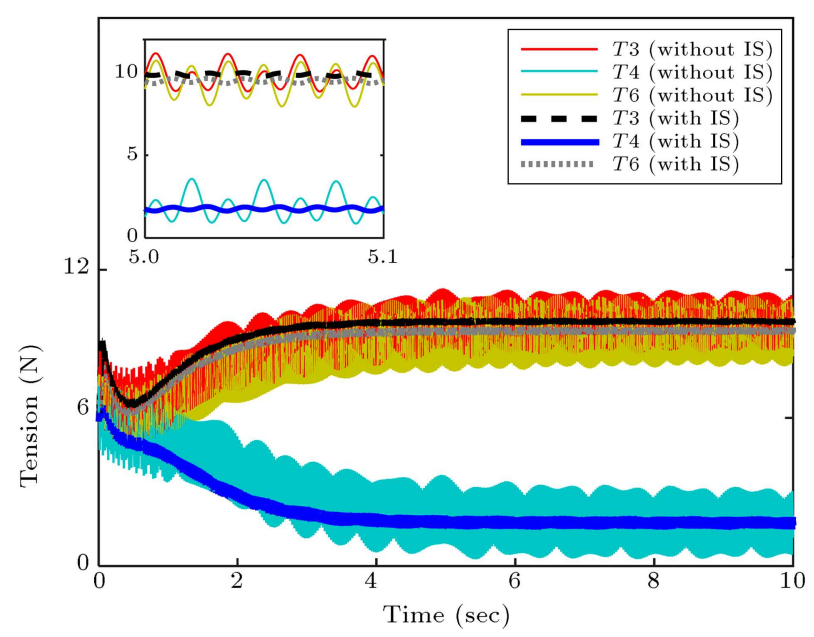

Figure 9. Tension of the 3rd, 4th, and 6th cables.

The trend of the distance between C.G. of the endeffector and the desired point towards the settling band of $1 \mathrm{~mm}$ is shown in Figure 10. It is shown that the time required to enter the settling band is decreased by $36 \%$ using input shaping method. The minimum values of the tensions in the second simulation are still higher, to some extent, than the minimum values in the first simulation. Therefore, the control gains in the second simulation can be further increased up to the values that the minimum tensions in both simulations are the same. This indicates that the time required to reach the settling band can be further decreased. In Figure 11, convergence of the 3rd Euler angle, $\varphi$, to the desired set-point is shown for both cases of with and without applying the input shaping method. It is shown that, using the input shaping approach, the settling time is reduced significantly. The control torques of the actuators are shown in Figures 12-14.

\subsection{Vibration reduction}

To investigate the effectiveness and contribution of the proposed approach, when the robot experiences high

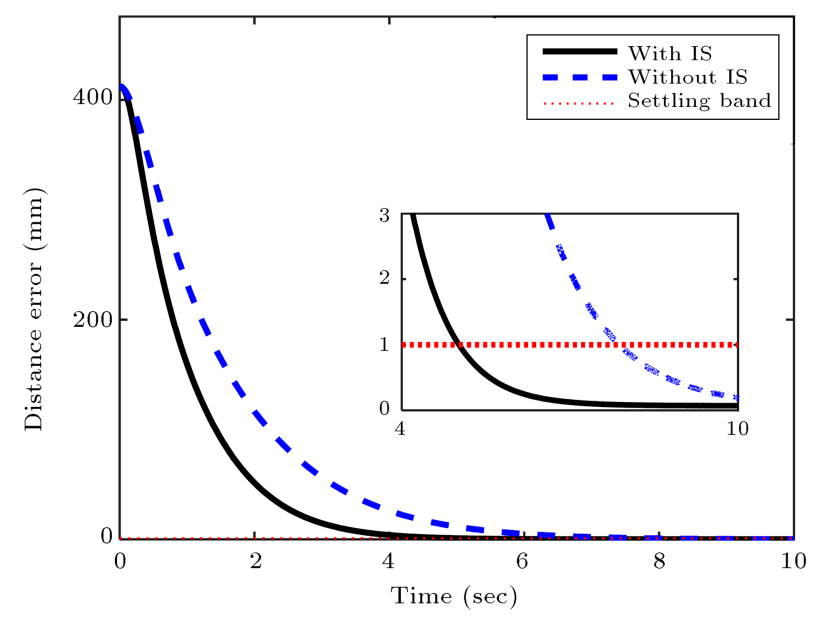

Figure 10. The distance between C.G. of the end-effector and the desired point.

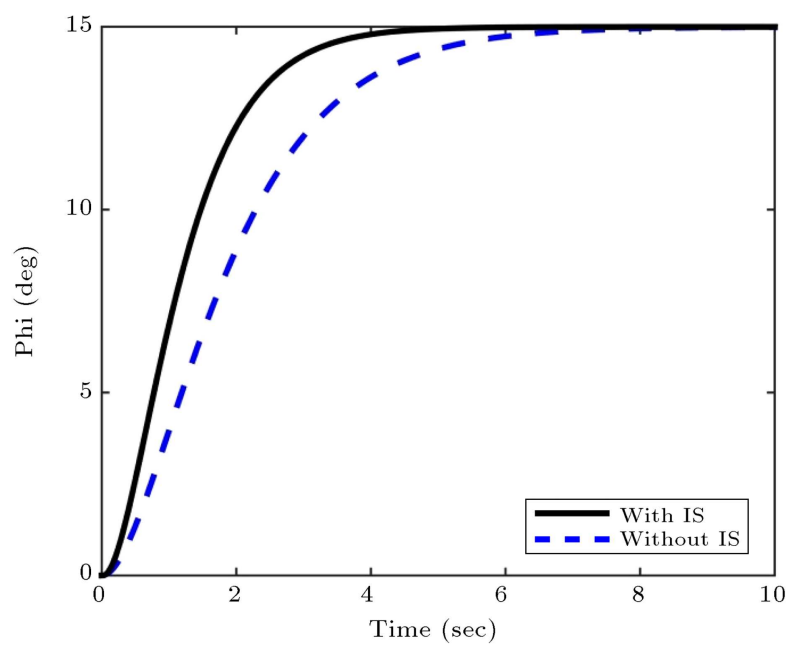

Figure 11. Convergence of the 3rd Euler angle, $\varphi$.

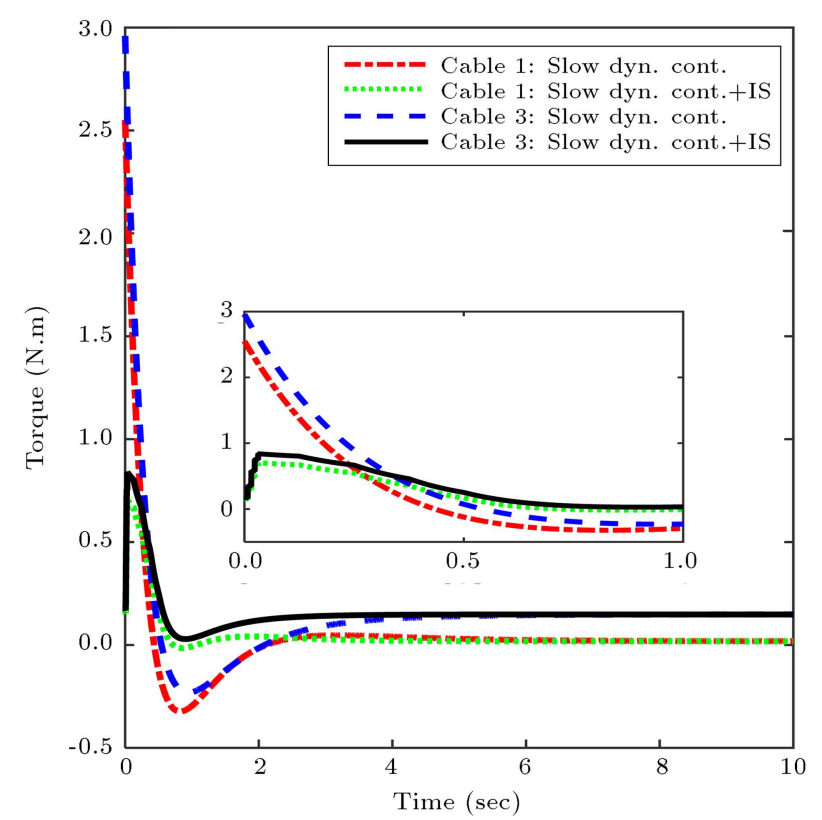

Figure 12. Control torque in the 1 st and 3rd actuators. 


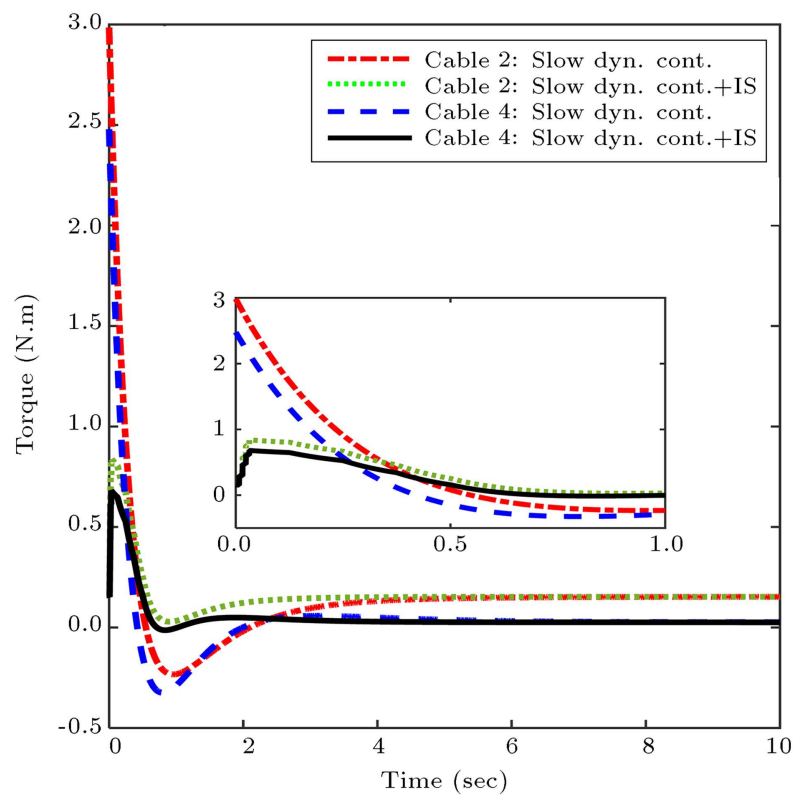

Figure 13. Control torque in the 2nd and 4th actuators.

cable flexibility, another case study with low stiffness of $E A=1.5 \mathrm{e} 3$ is performed. Similar to the previous case study, two simulations are performed for this case study. The desired path, in this case study, forms a square:

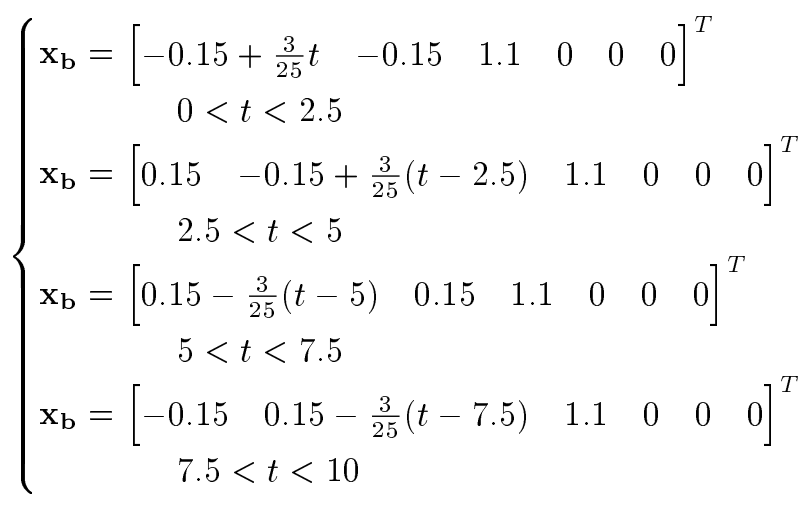

The vibration reduction using input shaping is investigated using the same control gains. Therefore, considering that the settling time is not important in this simulation, the control gains only need to result in a non-zero cable tension. This result can be achieved using the control gains as follows:

$$
\left\{\begin{array}{l}
\mathbf{K}_{\mathbf{p}}=\operatorname{diag}(4.25,4.25,21.25,0.4250 .425,4.25) \\
\mathbf{K}_{\mathbf{d}}=2 \sqrt{\mathbf{K}_{\mathbf{p}}}
\end{array}\right.
$$

The first simulation is carried out without using input shaping method. Considering the justification provided in the previous case study, one can obtain the natural frequencies of the system by calculating the FFT of the Euler angles and the cables tension (Figures 15 and 16).

As shown in Figures 15 and 16, the dominant modes occur at frequencies of $2.4 \mathrm{~Hz}, 17 \mathrm{~Hz}$, and

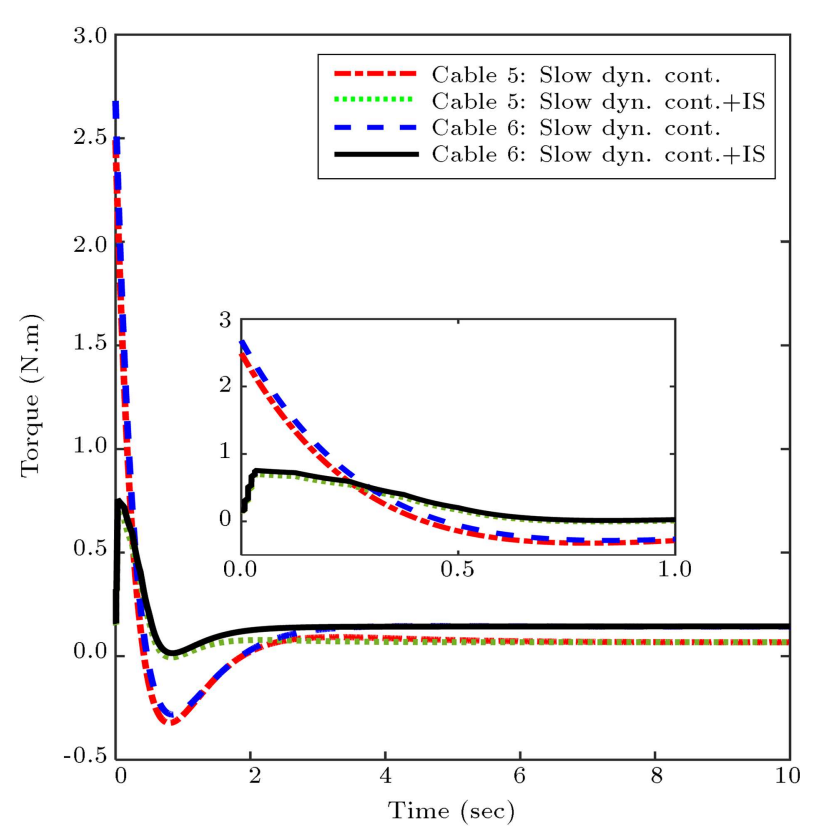

Figure 14. Control torque in the 5 th and 6 th actuators.

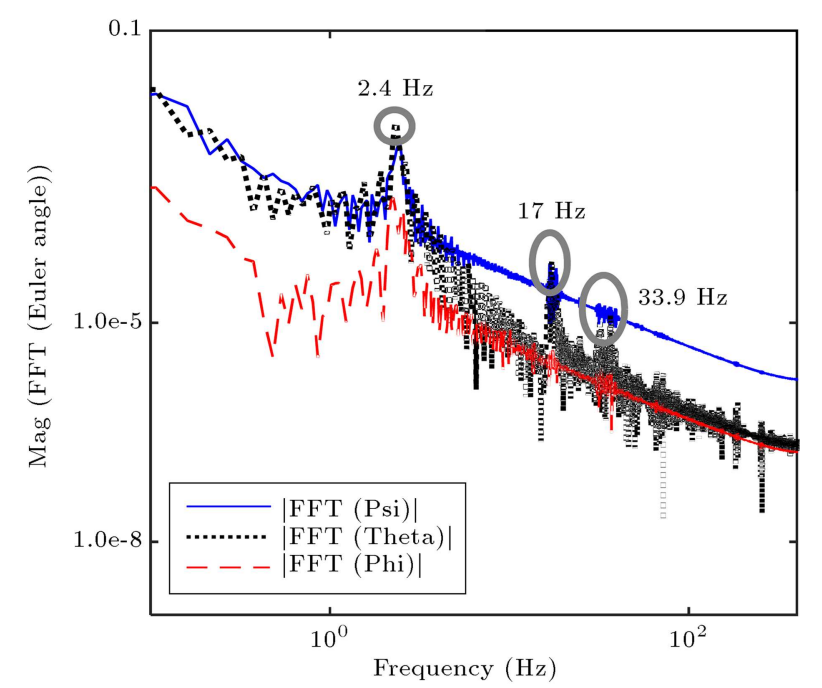

Figure 15. Frequency analysis of Euler angles.

$33.9 \mathrm{~Hz}$. Considering these frequencies, as well as $V_{\text {tol }}=5 \%$ and $\zeta=0$, the second simulation with a three-hump EI input shaper is performed. Figures 17 and 18 show the end-effector path in the horizontal plane and vertical direction, respectively. It is shown that the controller with input shaping significantly reduces the vibration of the end-effector. In Figure 19, the norm of Euler angles error is shown. The control torques are illustrated in Figures 20 and 21.

\subsection{Comparative study}

In order to further investigate the effectiveness of the proposed approach in vibration reduction, the last case study makes a comparison with a composite controller, which uses the feedback from the pose and velocity of the end-effector. In this case study, three simulations 


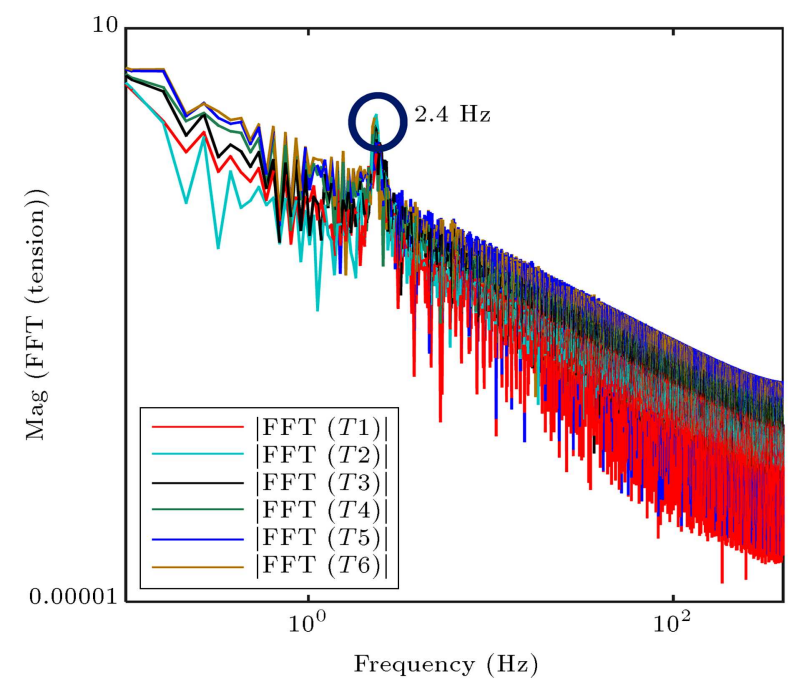

Figure 16. Frequency analysis of cables tension.

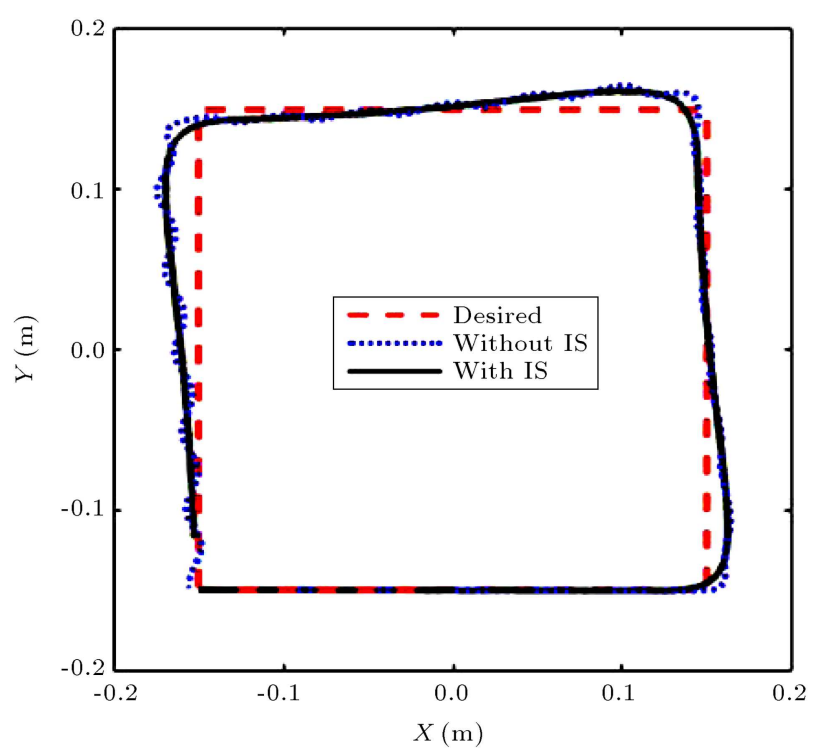

Figure 17. End-effector path in $X Y$ plane.

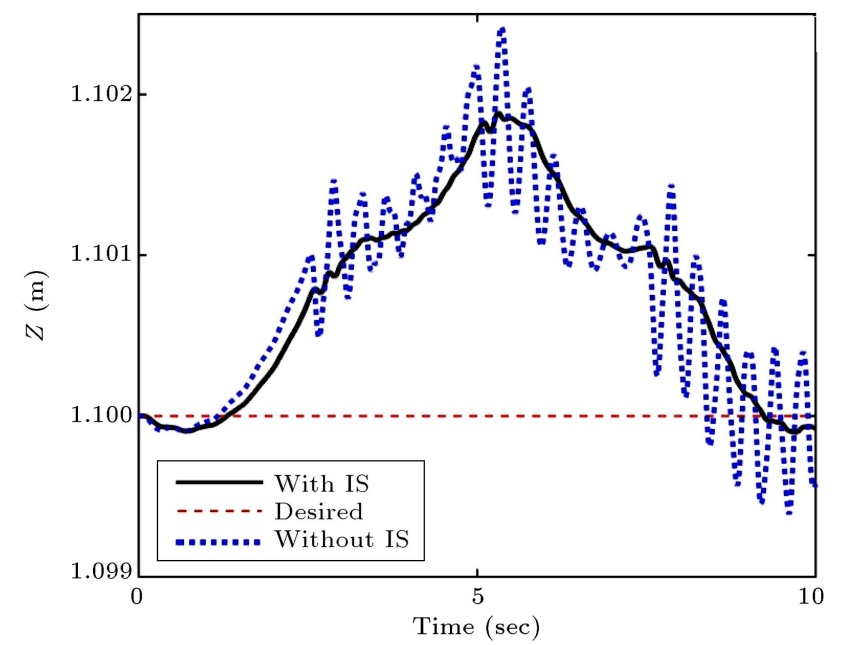

Figure 18. End-effector displacement in vertical direction.

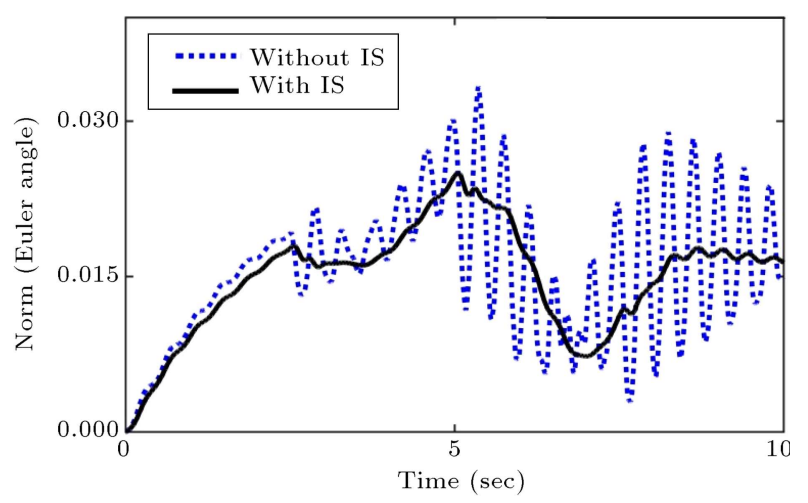

Figure 19. Norm of Euler angles error.

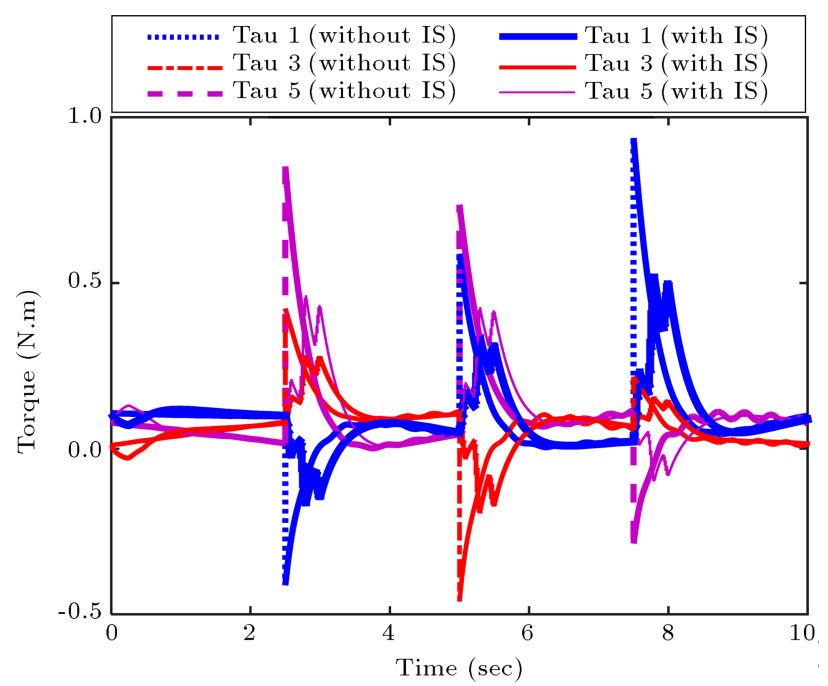

Figure 20. The torques $\tau_{1}, \tau_{3}$, and $\tau_{5}$.

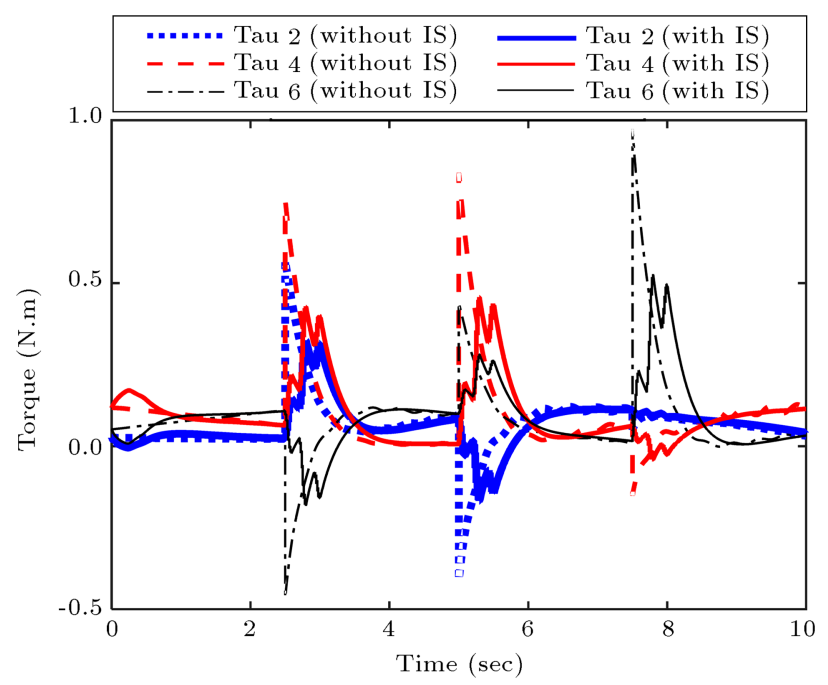

Figure 21. The torques $\tau_{2}, \tau_{4}$, and $\tau_{6}$.

are performed. In the first simulation, the input shaping method is not applied. The natural frequencies obtained from the first simulation results are used when applying the input shaping method for the second simulation. The third simulation is carried out using 
a composite controller without applying input shaping. The performance of the proposed method is compared with that of the composite controller using a new index.

One of the indices in determining the percentage of remaining vibration is Percentage of Residual Vibration (PRV), which is defined in Eq. (45). This index is only well defined for single mode LTI systems and difficult to measure from simulation data of multimode and nonlinear systems. In [27], the index PRE is defined as the ratio of the remaining energy in a system with input shaping to the remaining energy in a system without input shaping, where, for single mode condition, PRV $=\sqrt{\mathrm{PRE}}$. Since the index PRV is defined for single-mode LTI systems, this latter definition can be generalized to the multi-mode nonlinear systems with $\overline{\mathrm{PRV}}=\sqrt{\mathrm{PRE}}$. In this paper, to investigate the vibration reduction of the end-effector, a modified index of $\overline{\mathrm{PRV}}$ is defined. The new index is the square root of the ratio between the mean value of remaining kinetic energy with input shaping and the mean value of remaining kinetic energy without input shaping. The kinetic energy of the end-effector is calculated after entering a given settling band.

Cable stiffness of this case study is $E A=1.5 \mathrm{e} 3$. The simulations with point-to-point command are carried out for the following initial and desired points:

$$
\begin{aligned}
& \mathbf{x}_{\mathbf{0}}=\left[\begin{array}{llllll}
0 & 0 & 1.1 & 0 & 0 & 0
\end{array}\right]^{T}, \\
& \mathbf{x}_{\mathbf{f}}=\left[\begin{array}{llllll}
0.2 & 0.2 & 0.8 & 0 & 0 & \frac{\pi}{12}
\end{array}\right]^{T} .
\end{aligned}
$$

Considering an assumed settling time and error band for the rigid model, the control gains are obtained as follows:

$$
\begin{aligned}
& \mathbf{K}_{\mathbf{p}}=\operatorname{diag}(0.5,0.5,2.5,0.05,0.05,0.5), \\
& \mathbf{K}_{\mathbf{d}}=2 \sqrt{\mathbf{K}_{\mathbf{p}}} .
\end{aligned}
$$

Similar to the case study in Section 6.3, the natural frequencies and the parameters of the input shaper are obtained from the first simulation results. The second simulation is performed using the three-hump EI input shaping method. The third simulation is carried out using a composite controller, where the control gains for the slow dynamic are similar to those in the former simulations. Increasing the fast dynamic gain, $\mathbf{K}_{\delta}$, leads to an increased delay in reaching the settling band, while decreasing $\mathbf{K}_{\delta}$ results in an increase in vibration. For a fair comparison between the composite control approach and the proposed algorithm, fast dynamic gain of $\mathbf{K}_{\delta}=0.65 \mathbf{I}_{6}$ is selected, which results in the same delay time in the two simulations. Figures 22 and 23 show the first and the second control torques. Figure 24 shows the distance between C.G.

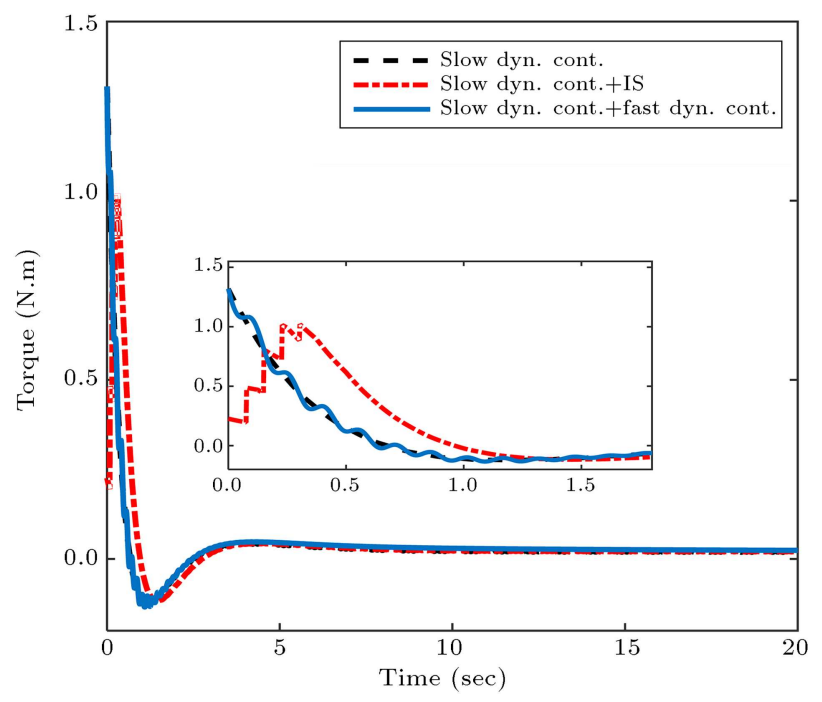

Figure 22. The control torque $\tau_{1}$.

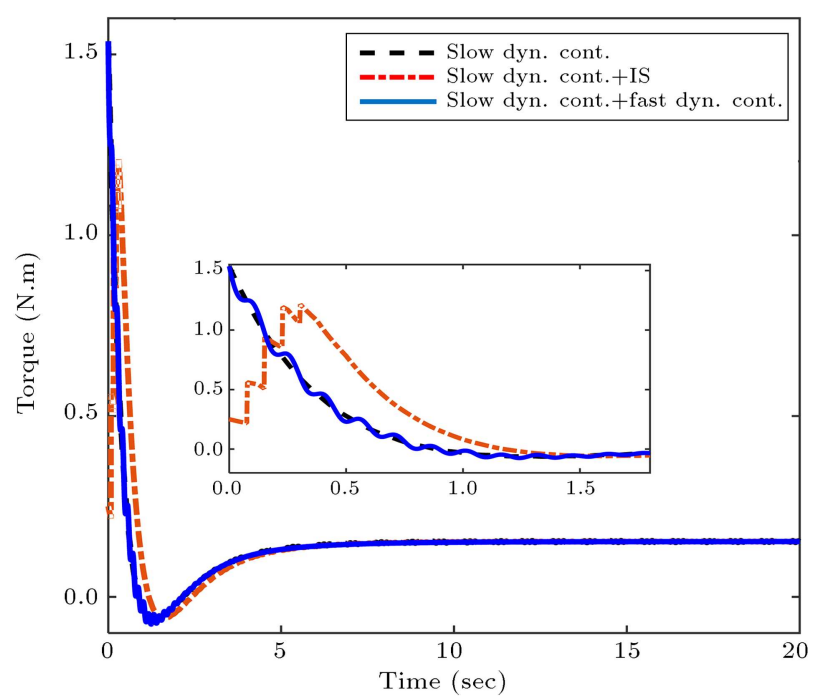

Figure 23. The control torque $\tau_{2}$.

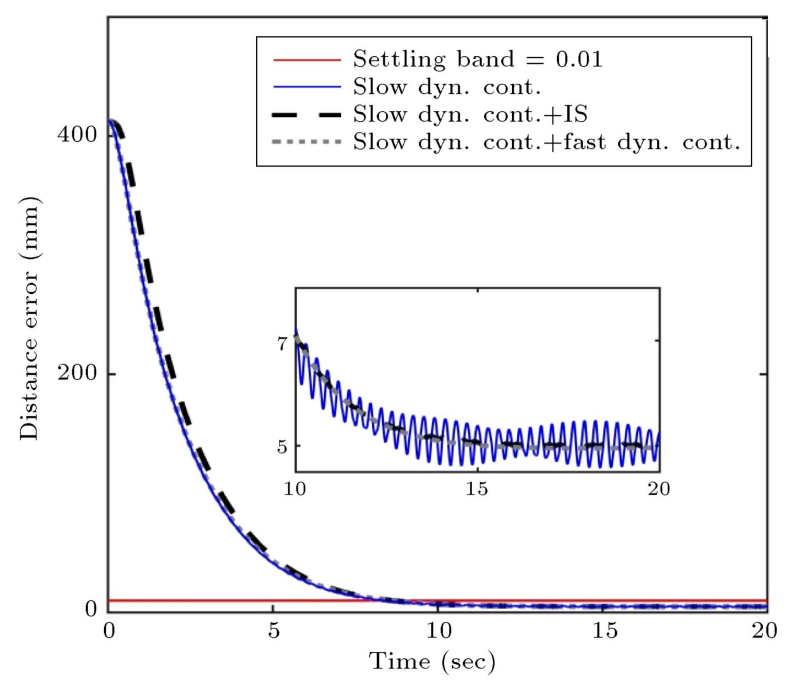

Figure 24. The distance between C.G. of the end-effector and the desired point. 
of the end-effector and the desired point for the three simulations. The index $\overline{\mathrm{PRV}}$ for the two simulations, a) slow dynamic controller with input shaping and b) slow dynamic controller plus fast dynamic controller, is $7.7 \%$ and $5.8 \%$, respectively, which shows that both suppressed the vibration effectively. However, the composite controller reduced the amplitude of the endeffector vibration slightly better than the proposed approach did at the cost of additional accurate endeffector feedback.

\section{Experimental results}

Verification of the approach presented in this paper is performed using ICasBot, a 6DOF cable-suspended robot manufactured in the robotic lab of Iran University of Science and Technology (Figure 25). The controller of the robot is implemented in the LabVIEW software, where it takes the feedback from incremental encoders installed on the output shaft of the motors (see the block diagram in Appendix C). The feedback is then mapped in the Cartesian space using the direct kinematic equation. Data communication between the LabVIEW and the hardware, i.e. encoders and motor drivers, is performed using two data cards (PCI 1780, Advantech). After processing and computation of the feedback signals in the LabVIEW, the PWM signals are generated and applied to the motors. The rest of the components of the experimental setup are described in $[28]$.

The following equation reflects the electrical relation between the torque $\tau$ in the output shaft of a DC motor and the applied voltage $\mathrm{V}$ :

$$
\begin{aligned}
& \tau=k_{1} v+k_{2} \dot{\beta}, \\
& k_{1}=\frac{n k_{t}}{R}
\end{aligned}
$$

$$
k_{2}=-n^{2} \frac{k_{t} k_{b}}{R}
$$

where $R$ is the motor winding resistance, $K_{b}$ is the back electromagnetic force constant, and $K_{t}$ denotes the motor torque constant. In addition, $n$ and $\dot{\beta}$ are the motor gear ratio and the output shaft speed, respectively. Considering Eq. (52) and the rigid dynamic equation, the torque $\tau$ can be omitted and the rigid dynamic equation with the input as voltage, $\mathbf{v}$, is obtained:

$$
\overline{\mathbf{M}} \ddot{\mathbf{x}}+\overline{\mathbf{C}}_{\mathbf{e}}+\overline{\mathbf{G}}=\overline{\mathbf{F}}_{\mathbf{e}} \mathbf{v}
$$

where $\overline{\mathbf{C}}_{\mathbf{e}}=\overline{\mathbf{C}}-\overline{\mathbf{F}} k_{2} \dot{\boldsymbol{\beta}}$ and $\overline{\mathbf{F}}_{\mathbf{e}}=\overline{\mathbf{F}} k_{1}$.

The control effort can be obtained using Eq. (39), considering that $\overline{\mathbf{C}}, \overline{\mathbf{F}}$, and $\boldsymbol{\tau}$ are replaced by $\overline{\mathbf{C}}_{\mathbf{e}}$, $\overline{\mathbf{F}_{\mathbf{e}}}$, and $\mathbf{v}$. In this work, the applied PWM signals to the motors are generated by Pulse Width Modulation of $12 \mathrm{v}$ supply. Noting that the DC voltage applied to the motor is the average value of the PWM signal, the applied PWM can be obtained from $\mathbf{P W M}(\%)=\frac{\mathbf{v}}{\mathbf{0 . 1 2}}$.

Since the cable robot used for the experiment has short cable length, the natural frequencies of the system are high. The smallest impulse time in the input shaping method is half the natural period of the system. Therefore, considering the Nyquist sampling theorem, vibration reduction using the input shaping method requires the sampling rate of the implemented controller to be theoretically four times the lowest natural frequency of the system. However, in the implementation, far higher sampling rate is needed. The sampling frequency of the implemented approach in the LabVIEW is in the range of [91$100 \mathrm{~Hz}$. In order to verify the approach using this sampling frequency, the system natural frequencies are intentionally lowered. This is performed by inserting springs with the same stiffness between each of the

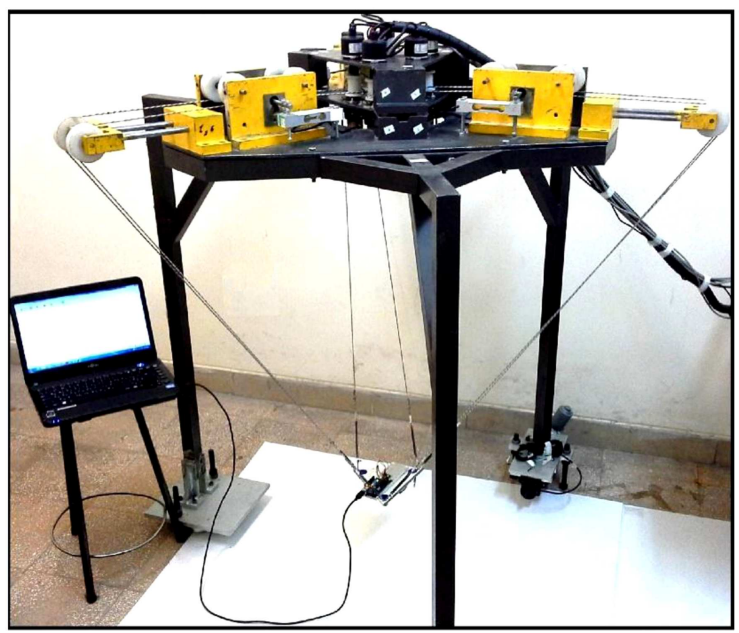

(a)

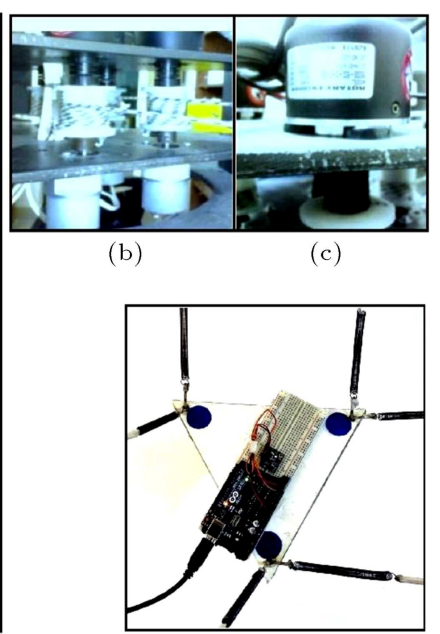

(d)

Figure 25. (a) The designed ICASBOT, (b) Motors, (c) Encoders, and (d) IMU, springs and end-effector. 
Table 2. Control inputs.

\begin{tabular}{lc}
\hline Initial position & {$[0,0,1.1 \mathrm{~m}, 0,0,0]$} \\
Final position & {$[0,0,0.8 \mathrm{~m}, 0,0,0]$} \\
Proportional gain & $K_{p}=\left[\begin{array}{cc}1.25,1.25,6.25,0.125,0.125,0.125] \\
\text { Derivative gain } \\
\text { Electrical parameters of motors }\end{array}\right.$ \\
$\begin{array}{c}K_{d}=2 \times \sqrt{(K p)} \\
\text { Cable stiffness }\end{array}$ & $\begin{array}{c}\text { (N.m/V) }, k_{2}=-0.08 \text { (N.m.sec) } \\
440 \mathrm{~N} / \mathrm{m}\end{array}$ \\
\hline
\end{tabular}

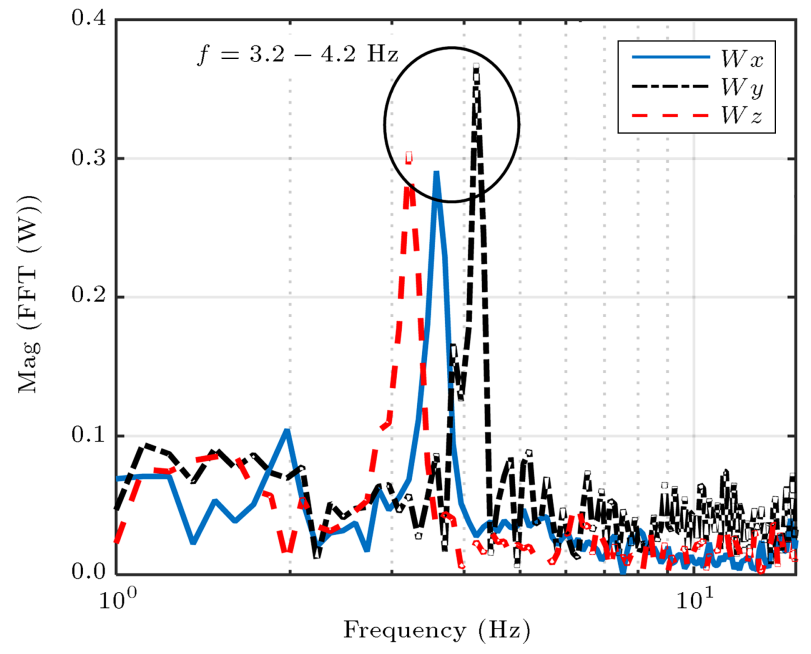

Figure 26. Frequency analysis of the angular velocity in the experiment.

cables and the end-effector. The end-effector vibration during the tracking is measured using an IMU sensor, GY86-MPU 6050. This sensor measures the linear accelerations as well as the angular velocities in three orthonormal directions. The experimental parameters are provided in Table 2. Two tests are carried out in this study.

\subsection{Verifying the dynamic model}

In the first experiment, a comparison between the frequency spectrum of the simulation and the test verifies the derived dynamic model. A point-to-point vertical motion of the end-effector from $z=1.1 \mathrm{~m}$ to $z=0.8 \mathrm{~m}$ is performed. As shown in Figure 26, the dominant vibrational frequencies of the system, obtained from the IMU, range between [3.2-4.2 Hz]. Next, a simulation with the same cable stiffness as that in the experiment is performed. Figure 27 represents the frequency spectrum of the cable tension. As shown, the frequency of the flexible model is $3.75 \mathrm{~Hz}$, which well matches the test results.

\subsection{Investigation of the vibration reduction approach}

In the second experiment, the effectiveness of the input shaping method in vibration reduction is investigated. The trajectory of the end-effector is considered as follows:

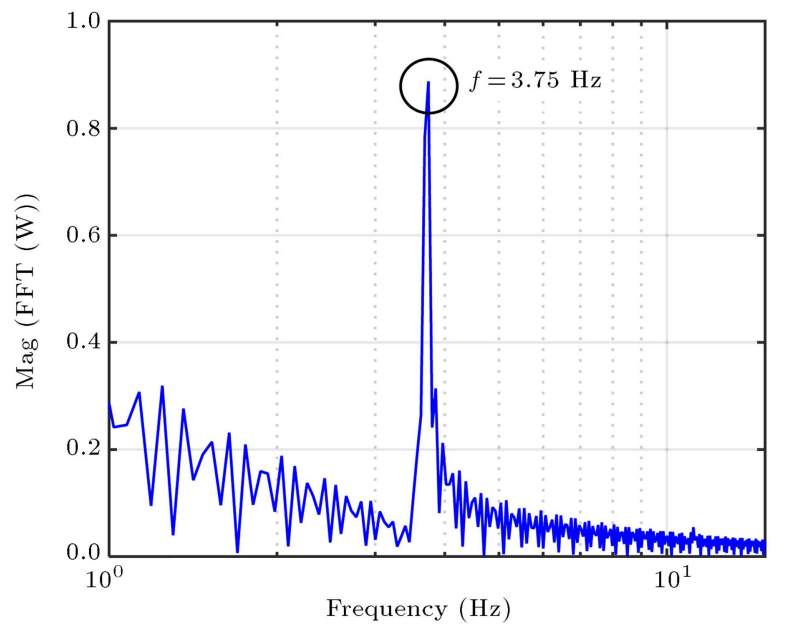

Figure 27. Frequency analysis of the cable tension in the simulation.

$$
\begin{aligned}
\mathbf{x}_{\mathbf{b}}= & {\left[0.1 \sin \left(\frac{2 \pi}{15} t\right) 0.1 \cos \left(\frac{2 \pi}{15} t\right) 1.1\right.} \\
& \left.+0.01 \cos \left(\frac{4 \pi}{3} t\right) \quad \mathbf{0}_{\mathbf{1} \times \mathbf{3}}\right]^{T} .
\end{aligned}
$$

In this experiment, the trajectory tracking is performed for two cases, i.e. with and without applying the input shaping method. In the case without input shaping, the frequency analysis shows that the dominant natural frequencies are in the range of [2.7-4.3 Hz]. Therefore, for the second test, the parameters of the input shaping method are obtained considering the middle frequency of $3.5 \mathrm{~Hz}$. Figures 28-30 compare the angular velocities in the two tests obtained from the IMU sensor. Considering the data in Figures 28-30, the ratio of the standard deviation of the test with input shaping to that of the test without input shaping is in the range of [0.22-0.24]. Indeed, this index indicates the reduction ratio of the vibration amplitude.

Figures 31-33 show the frequency spectrum of the angular velocities. Comparing the test results indicates that the input shaping method significantly reduced the vibration of the main modes. It is notable that the frequency content of the test data, obtained from the sensor, includes the unwanted data related to noise and disturbances in addition to the vibration data of the system. Since the noise and the disturbances in the 


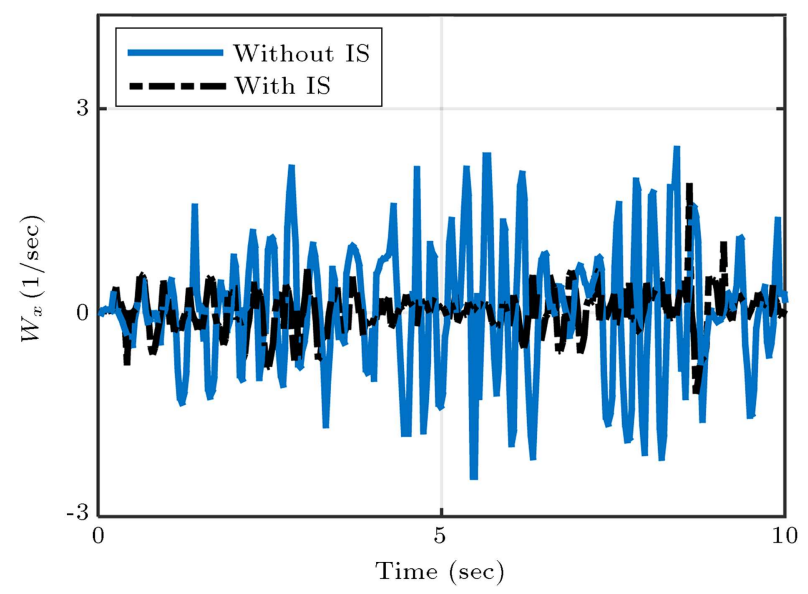

Figure 28. Angular velocity $\omega_{x}$ obtained from IMU.

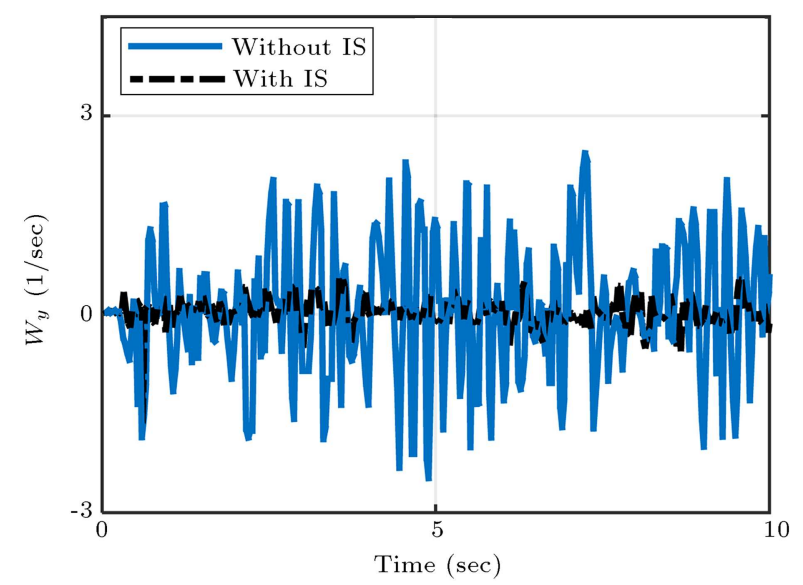

Figure 29. Angular velocity $\omega_{y}$ obtained from IMU.

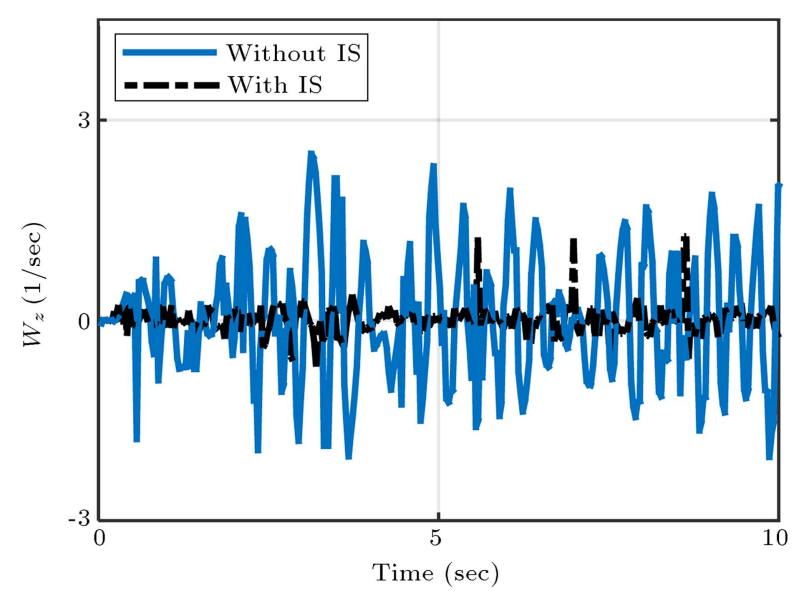

Figure 30. Angular velocity $\omega_{z}$ obtained from IMU.

system are distributed over the whole range of the frequency spectrum, beyond the range of the vibrational modes of the system, the vibration reduction algorithm has no remarkable effect.

The maximum vibrational frequency is nearly $4.5 \mathrm{~Hz}$. Therefore, considering the input shaping method, the sampling rate is about 5 times the required

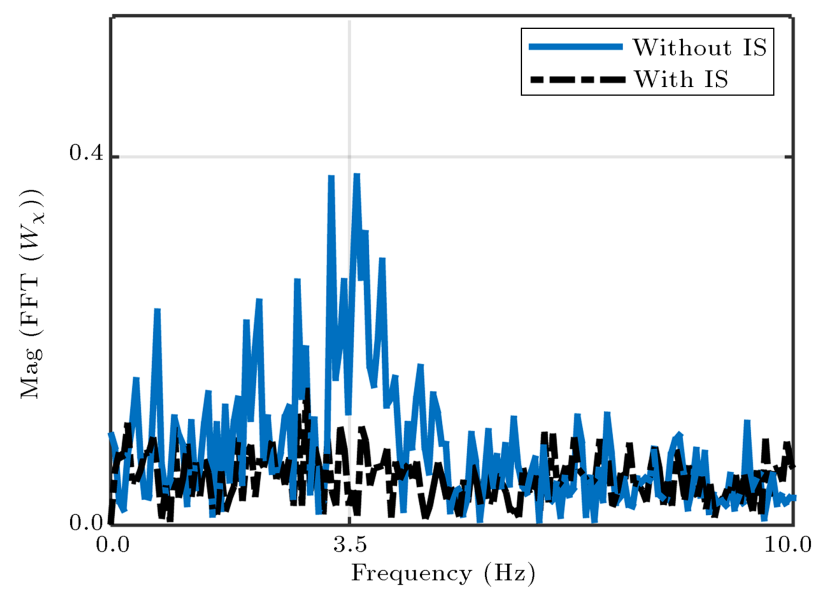

Figure 31. Frequency analysis of the angular velocity $\omega_{x}$.

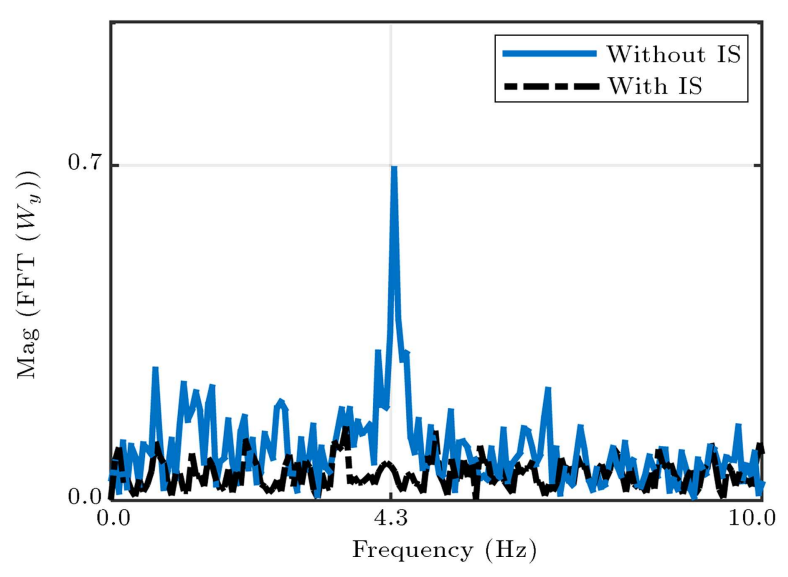

Figure 32. Frequency analysis of the angular velocity $\omega_{y}$.

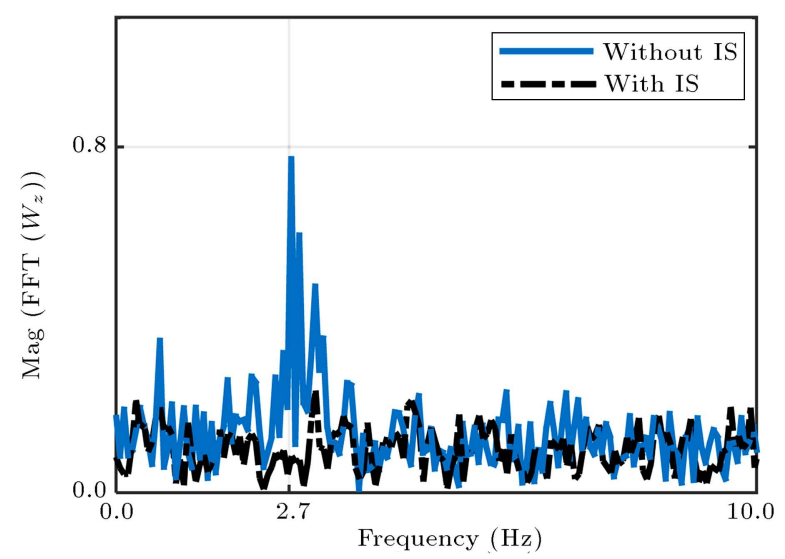

Figure 33. Frequency analysis of the angular velocity $\omega_{z}$.

value according to the Nyquist theorem. In the implementation, this is the minimum required sampling rate in order to apply the impulses of the input shaping method at the proper time. However, in the industrial implementation, higher sampling rate can be achieved, making it possible to suppress the residual vibration at higher frequencies. The control inputs of the test are shown in Figures 34 and 35. 


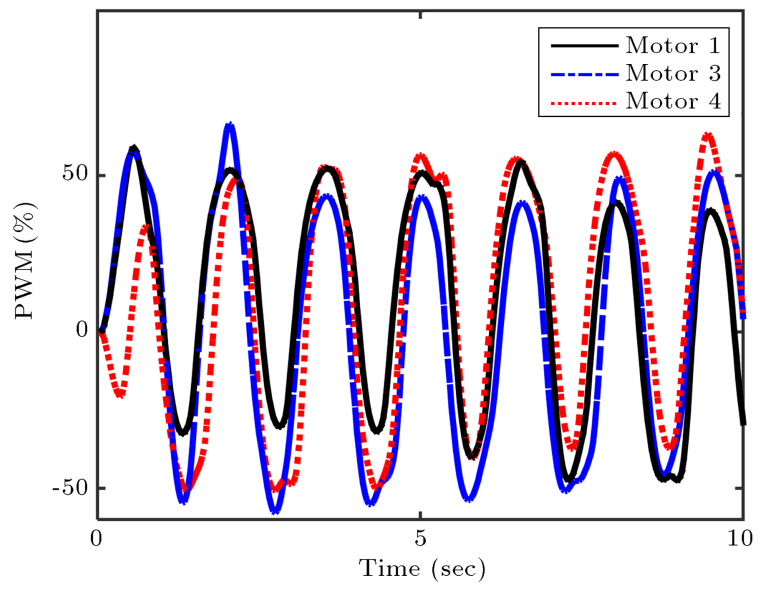

Figure 34. The control input of the 1st, 3rd, and 4th motors.

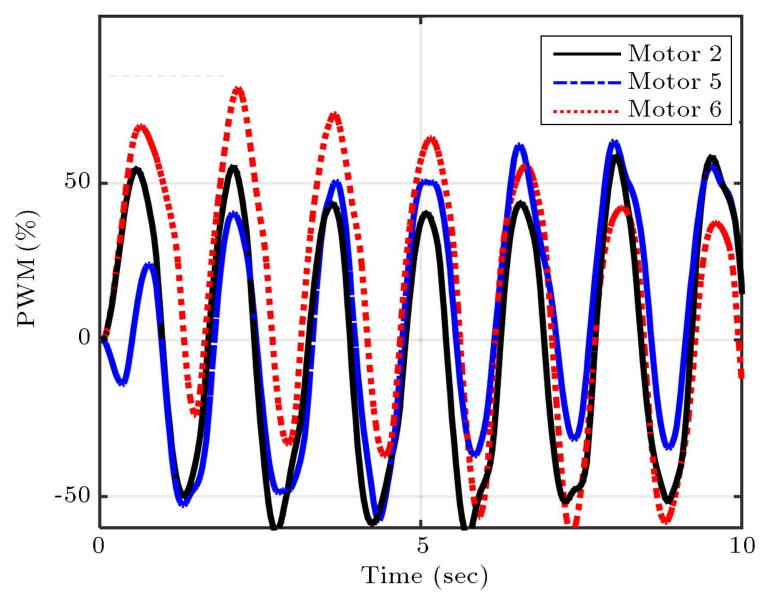

Figure 35. The control input of the 2 nd, 5 th, and 6 th motors.

\section{Conclusions}

In this paper, dynamic equations of a 6DOF cablesuspended robot with elastic cables are derived employing Gibbs-Appel formulation. After comparing three control approaches, feedback linearization method based on the rigid cable model and feedback of the actuators was selected. To suppress the vibration caused by flexibility of cables, three-hump EI input shaper was employed to modify the desired input of the end-effector in Cartesian space such that the natural modes of the system were not excited. Several case studies were carried out in the simulation section. The first case study showed that the trajectory of the cable robot with elastic cables converged to the trajectory of the one with rigid cable model. The second and third case studies showed two contributions of using input shaping for conditions of low and high cable flexibility. When cable flexibility was high, the simulation indicated that the input shaping had a noticeable effect in vibration reduction of the endeffector. In the case of low cable flexibility, vibration of the end-effector was not significant and the proposed approach contributed to the reduction in the time required to reach the desired track. The last simulation compared the proposed approach with a composite controller. The composite controller comprised a slow dynamic term for controlling the overall motion of the end-effector using the feedback from the motor encoder and a fast dynamic term to suppress the end-effector vibration using the feedback from the end-effector pose and velocity. It was demonstrated that the composite controller had slightly better performance in vibration reduction at the cost of additional sensors and more controller computations. Finally, the experiment on a laboratory robot manufactured in Iran University of Science and Technology, i.e. ICastBot, demonstrated the effectiveness of the proposed approach in vibration reduction. In the experiment, the vibrations were measured employing an IMU sensor attached to the endeffector for specifying the system natural frequencies and investigating the vibration reduction.

\section{Acknowledgments}

The authors gratefully acknowledge the support of INSF under the grant contracts $93-\mathrm{S}-39125$.

\section{Nomenclature}

A

$\mathbf{A}_{\mathbf{i}}, i=1 \ldots 4$

Cross section area of the cables

C

Coefficient matrices

Coriolis and centrifugal vector of the flexible system

$\mathbf{C}_{\mathbf{d}} \quad$ Cable damping coefficient

$c_{d} \quad$ Cable damping coefficient per unit

$E \quad$ Young's modulus of elasticity

$\mathbf{e}=\mathbf{x}_{\mathbf{d}}-\mathbf{x} \quad$ End-effector pose error

G Gravity vector of the flexible system

$\mathbf{I}_{\mathbf{e}} \quad$ Moment of inertia tensor of the end-effector

$\mathbf{I}_{\mathbf{m}} \quad$ Equivalent moment of inertia of the rotating parts of the motors

$\mathbf{K}_{\boldsymbol{\delta}} \quad$ The gain of the torque for stabilizing the fast dynamic

$\mathbf{K}_{\mathbf{p}}, \mathbf{K}_{\mathbf{d}} \quad$ Proportional and derivative gains of the feedback linearization controller

$L_{\text {mult }} \quad$ The composite shaped impulse sequence

M Symmetric positive definite mass matrix of the flexible system

$m_{e} \quad$ End-effector mass

$\mathbf{P} \quad$ Euler angles rate matrix

PWM Pulse width modulation 


\section{References}

1. Diao, X. and Ma, O. "Vibration analysis of cabledriven parallel manipulators", Multibody Syst. Dyn., 21(4), pp. 347-360 (2009).

2. Korayem, M.H., Tourajizadeh, H. and Bamdad, M. "Dynamic load carrying capacity of flexible cable suspended robot: robust feedback linearization control approach", J. Intell. Robot. Syst., 60(3), pp. 341-363 (2010).

3. Korayem, M.H., Taherifar, M. and Tourajizadeh, H. "Compensating the flexibility uncertainties of a cable suspended robot Using SMC approach", Robotica, 33(3), pp. 578-598 (2015).

4. Laroche, E., Chellal, R., Cuvillon, L. and Gangloff, J. "A preliminary study for control of parallel cabledriven manipulators", In Cable-Driven Parallel Robots, 12, pp. 353-369, Springer, Berlin, Heidelberg (2013).

5. Meunier, G., Boulet, B. and Nahon, M. "Control of an overactuated cable-driven parallel mechanism for a radio telescope application", IEEE Trans. on Control Systems Technology, 17(5), pp. 1043-1054 (2009).

6. Caverly, R.J. and Forbes, J.R. "Dynamic modeling and noncollocated control of a flexible planar cable-driven manipulator", IEEE Trans. on Robotics, 30(6), pp. 1386-1397 (2014).

7. Shao, Z.F., Tang, X., Wang, L.P. and Chen, X. "Dynamic modeling and wind vibration control of the feed support system in FAST", Nonlinear Dyn., 67(2), pp. $965-985$ (2012).

8. Liu, Z., Tang, X. and Wang, L. "Research on the dynamic coupling of the rigid-flexible manipulator", Robot. Comput. Integr. Manufac., 32, pp. $72-82$ (2015).

9. Khosravi, M.A. and Taghirad, H.D. "Dynamic analysis and control of fully-constrained cable robots with elastic cables: variable stiffness formulation", In CableDriven Parallel Robots, Springer International Publishing, pp. 161-177 (2015).

10. Ozgoli, S. and Taghirad, H.D. "A Survey on the control of flexible joint robots", Asian Journal of Control, 8(4), pp. 332-344 (2006).

11. Singer, N., Singhose, W. and Seering, W. "Comparison of filtering methods for reducing residual vibration", European Journal of Control, 5(2-4), pp. 208-218 (1999).

12. Luo, L., Wang, S., Mo, J. and Cai, J. "On the modeling and composite control of flexible parallel mechanism", Int. J. Adv. Manuf. Technol., 29(7), pp. 786-793 (2006).

13. Hu, Q. "Robust integral variable structure controller and pulse-width pulse-frequency modulated input shaper design for flexible spacecraft with mismatched uncertainty/disturbance", ISA Transactions, 46(4), pp. 505-518 (2007). 
14. Zhang, Q., Mills, J.K., Cleghorn, W.L., Jin, J. and Zhao, C. "Trajectory tracking and vibration suppression of a 3-PRR parallel manipulator with flexible links", Multibody Syst. Dyn., 33(1), pp. 27-60 (2015).

15. Zhang, Q., Mills, J.K., Cleghorn, W.L., Jin, J. and Sun, Z. "Dynamic model and input shaping control of a flexible link parallel manipulator considering the exact boundary conditions", Robotica, 33(06), pp. 1201-1230 (2015).

16. Öltjen, J., Kotlarski, J. and Ortmaier, T. "Reduction of end effector oscillations of a parallel mechanism with modified motion profiles", IEEE 10th Conf. on Industrial Electronics and Applications, pp. 823-829 (2015).

17. Li, W.P., Luo, B. and Huang, H. "Active vibration control of flexible joint manipulator using input shaping and adaptive parameter auto disturbance rejection controller", JSV, 363, pp. 97-125 (2016).

18. Blackburn, D.F. "Command shaping for vibration reduction in nonlinear cabled systems", M.S. Thesis, School of Mechanical Engineering, Georgia Institute of Technology (2006).

19. Park, J., Kwon, O. and Park, J.H. "Anti-sway trajectory generation of incompletely restrained wiresuspended system", JMST, 27(10), pp. 3171-3176 (2013).

20. Thalapil, J. "Input shaping for sway control in gantry cranes", Journal of Mechanical and Civil Engineering, 1(2), pp. 36-46 (2012).

21. Garrido, S., Abderrahim, M., Gimenez, A. and Diez, R. "Anti-swinging input shaping control of an automatic construction crane", IEEE Trans. Autom. Sci. Eng., 5(3), pp. 549-557 (2008).

22. Elbadawy, A.A. and Shehata, M.M.G. "Anti-sway control of marine cranes under the disturbance of a parallel manipulator", Nonlinear Dyn., 82(1), pp. 415434 (2015).

23. Fatehi, M.H., Eghtesad, M. and Amjadifard, R. "Using singular perturbation method for controlling a crane system with a flexible cable and large swing angle", Journal of Low Frequency Noise, Vibration and Active Control, 34(3), pp. 361-383 (2015).

24. Kokotovic, P., Khalil, H.K. and O'Reilly, J., Singular Perturbation Methods in Control: Analysis and Design, 25, Siam (1999).

25. Singhose, W. "Command shaping for flexible systems: A review of the first 50 years", Int. J. Precis. Eng. Manuf., 10(4), pp. 153-168 (2009).

26. Vaughan, J., Yano, A. and Singhose, W. "Comparison of robust input shapers", JSV, 315(4), pp. 797-815 (2008).

27. Kozak, K., Ebert-Uphoff, I. and Singhose, W. "Locally linearized dynamic analysis of parallel manipulators and application of input shaping to reduce vibrations", J. Mech. Des., 126(1), pp. 156-168 (2004).
28. Korayem, M.H., Imanian, A., Tourajizadeh, H., Khayatzadeh, S., Maddah, S.M.E., Tajik, A. and Manteghi, S. "A method for simultaneous control of speed and torque of the motors of a cable suspended robot for tracking procedure", Scientia Iranica B, 20(5), pp. $1550-1565$ (2013).

\section{Appendix A}

The coefficient matrices in the kinematic and dynamic equations

The relation between angular velocity of the endeffector in the frame attached to the end-effector and the time derivative of the Euler angles is:

$$
\boldsymbol{\omega}=\mathbf{P} \dot{\boldsymbol{\psi}}, \quad \text { where }: \quad \dot{\boldsymbol{\psi}}=[\psi, \theta, \varphi]^{T}
$$

where $\mathbf{P}$ is Euler angle rate matrix. Therefore, the vectors $\dot{\tilde{\mathbf{x}}}$ and $\ddot{\tilde{\mathbf{x}}}$ can be rewritten as:

$$
\dot{\tilde{\mathbf{x}}}=\mathbf{A}_{\mathbf{3}} \dot{\mathbf{x}}, \quad \ddot{\tilde{\mathbf{x}}}=\mathbf{A}_{\mathbf{3}} \ddot{\mathbf{x}}+\mathbf{A}_{\mathbf{4}},
$$

where:

$$
\mathbf{x}=\left[\begin{array}{c}
\chi \\
\psi
\end{array}\right], \quad \mathbf{A}_{3}=\left[\begin{array}{cc}
\mathbf{I}_{3} & \mathbf{0}_{\mathbf{3}} \\
\mathbf{0}_{\mathbf{3}} & \mathbf{P}
\end{array}\right], \quad \mathbf{A}_{\mathbf{4}}=\left[\begin{array}{c}
\mathbf{0}_{\mathbf{3} \times \mathbf{1}} \\
\dot{\mathbf{P}} \dot{\psi}
\end{array}\right] .
$$

Considering the Jacobian matrix $\boldsymbol{\Lambda}$ as:

$$
\boldsymbol{\Lambda}=\left[\begin{array}{ccc}
\cdots & -{ }^{\mathbf{N}} \hat{\mathbf{q}}_{\mathbf{i}} & \ldots \\
\cdots & -\left({ }^{\mathbf{N}} \mathbf{R}_{\mathbf{B}} \mathbf{r}_{\mathbf{B i} \times} \times{ }^{\mathbf{N}} \hat{\mathbf{q}}_{\mathbf{i}}\right) & \ldots
\end{array}\right]_{6 \times 6}
$$

the matrices $\mathbf{A}_{1}$ and $\mathbf{A}_{2}$ are obtained:

$$
\mathbf{A}_{\mathbf{1}}=\frac{1}{r_{p}} \boldsymbol{\Lambda}^{\mathbf{T N}} \overline{\mathbf{R}}_{\mathbf{B}}
$$

where:

$$
\begin{aligned}
& { }^{\mathbf{N}} \overline{\mathbf{R}}_{\mathbf{B}}=\left[\begin{array}{cc}
\mathbf{I}_{\mathbf{3}} & \mathbf{0}_{\mathbf{3}} \\
\mathbf{0}_{\mathbf{3}} & { }^{\mathbf{N}} \mathbf{R}_{\mathbf{B}}
\end{array}\right], \\
& \mathbf{A}_{\mathbf{2}}=\frac{1}{r_{p}} \dot{\boldsymbol{\Lambda}}^{\mathbf{T}}{ }^{\mathbf{N}} \overline{\mathbf{R}}_{\mathbf{B}} \dot{\tilde{\mathbf{x}}},
\end{aligned}
$$

where ${ }^{\mathbf{N}} \hat{\mathbf{q}}_{\mathbf{i}}$ is the unit vector along the $i$ th cable, from motor side to the end-effector side, expressed in the inertial frame. ${ }^{\mathbf{N}} \mathbf{R}_{\mathbf{B}}$ is the rotation matrix of the frame attached to the end-effector with respect to the inertial frame.

\section{Appendix B}

\section{Feedback linearization of error dynamics for the flexible system}

After obtaining the 2nd derivative of Eq. (36) and replacing $\ddot{\boldsymbol{\beta}}$ with the expression obtained from the last six rows of Eq. (16), the following equation is obtained:

$$
\begin{aligned}
& \frac{\mathbf{I}_{\mathbf{m}}}{r_{p}}\left(\ddot{\tilde{\mathbf{M}}} \mathbf{x}^{(2)}+2 \dot{\tilde{\mathbf{M}}} \mathbf{x}^{(3)}+\tilde{\mathbf{M}} \mathbf{x}^{(4)}+\ddot{\tilde{\mathbf{C}}}+\ddot{\tilde{\mathbf{G}}}-\ddot{\mathbf{q}}\right) \\
& \quad+r_{p} \mathbf{K} \Delta \mathbf{q}=\boldsymbol{\tau}
\end{aligned}
$$




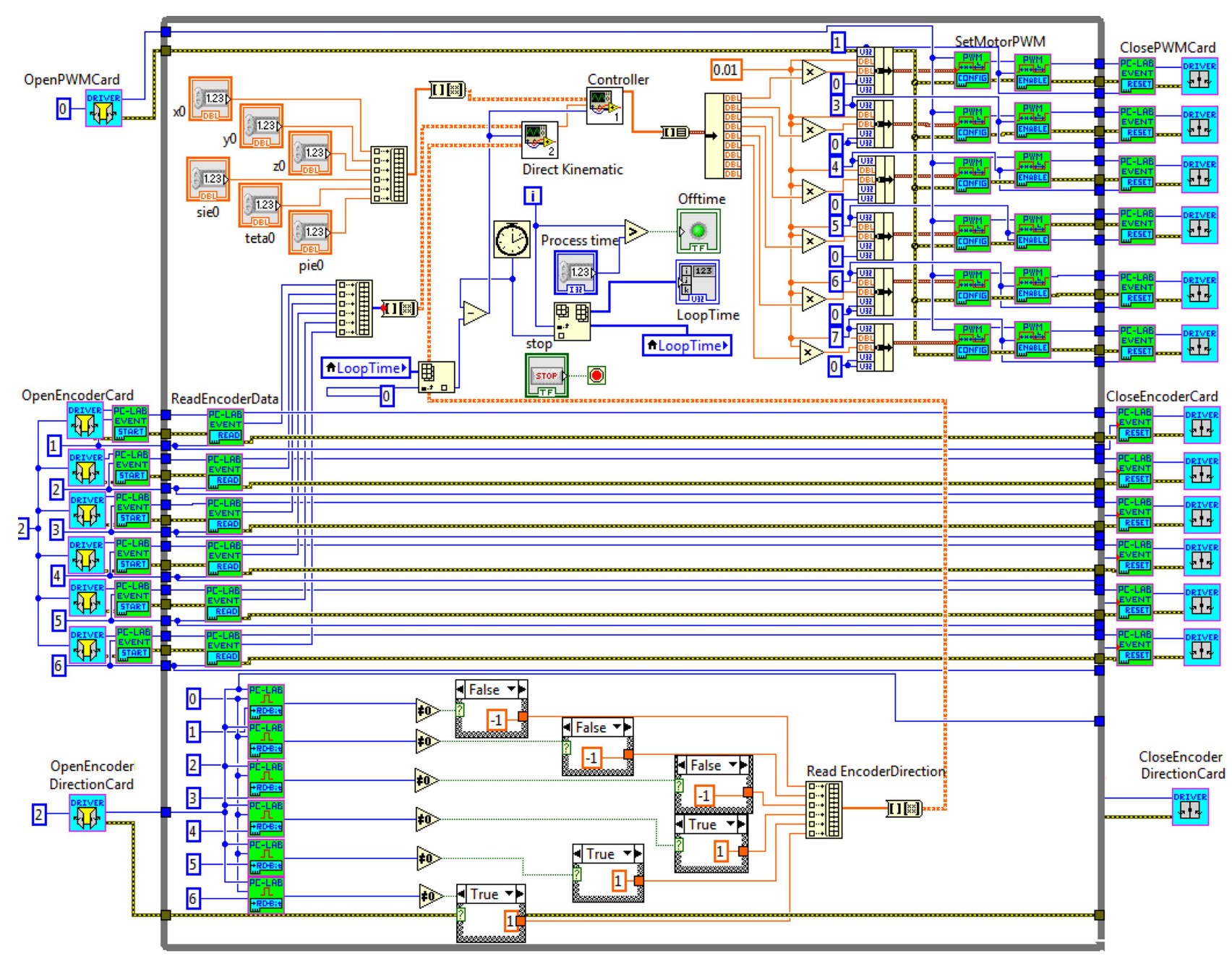

Figure C.1. LabVIEW block diagram.

where $\mathbf{x}^{(i)}$ denotes the $i$-th derivative of $\mathbf{x}$. Substituting the cable length deflection from Eq. (36) into Eq. (B.1) gives:

$$
\begin{aligned}
& \frac{\mathbf{I}_{\mathbf{m}}}{r_{p}}\left(\ddot{\tilde{\mathbf{M}}} \mathbf{x}^{(2)}+2 \dot{\tilde{\mathbf{M}}} \mathbf{x}^{(3)}+\tilde{\mathbf{M}} \mathbf{x}^{(4)}+\ddot{\tilde{\mathbf{C}}}+\ddot{\tilde{\mathbf{G}}}-\ddot{\mathbf{q}}\right) \\
& \quad+r_{p} \mathbf{K}(\tilde{\mathbf{M}} \ddot{\mathbf{x}}+\tilde{\mathbf{C}}+\tilde{\mathbf{G}})=\tau .
\end{aligned}
$$

If the control torque of Eq. (37) is inserted in Eq. (B.2), the linear error dynamics of the system are obtained:

$$
\mathbf{e}^{(4)}+k_{3} \mathbf{e}^{(3)}+k_{2} \mathbf{e}^{(2)}+k_{1} \dot{\mathbf{e}}+k_{0} \mathbf{e}=0 .
$$

\section{Appendix C:}

\section{Lab VIEW}

Figure C.1 shows the LabVIEW block diagram.

\section{Biographies}

Moharam Habibnejad Korayem was born in Tehran, Iran, in 1961. He received his BS (Hon) and
MS degrees in Mechanical Engineering from Amirkabir University of Technology in 1985 and 1987, respectively. He obtained his PhD degree in Mechanical Engineering from the University of Wollongong, Australia, in 1994. He is now Professor of Mechanical Engineering at Iran University of Science and Technology. His research interests include dynamics of elastic mechanical manipulators, trajectory optimization, symbolic modelling, robotic multimedia software, mobile robots, industrial robotics standard, robot vision, soccer robot, and the analysis of mechanical manipulator with maximum load carrying capacity.

Mahdi Yousefzadeh was born in Iran on December 22,1976 . He received his BSc in Mechanical Engineering from Babol Noshirvani University of Technology in 2000 and then obtained his MSc from K.N. Toosi University of Technology in 2003 in the field of automotive system design. He received his $\mathrm{PhD}$ degree in mechanical engineering from Iran University of Science and Technology in the field of dynamics, vibration and control. His research interests include 
dynamic, control and vibration analysis of robotic and automotive systems. He has a 14-year experience in machine design for automotive industry as well as four experience of teaching in different universities in Babol, Iran.

Soleiman Manteghi was born in Iran on March 20,
1980. He received his BSc in Electrical Engineering from Malek-e-Ashtar University of Technology in 2003. In 2013, he obtained his MSc degree in Computer and Mechatronics Engineering from Islamic Azad University Branch of Science \& Research. His research interest is in the mechatronic fields particularly robotic systems. 